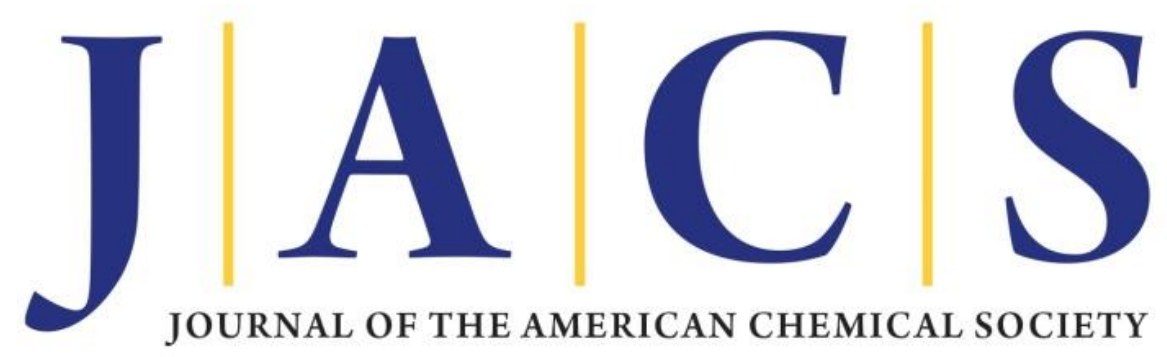

Supplemental Information for

\title{
Single-Crystal Polycationic Polymers Obtained by Single-Crystal-to- Single-Crystal Photopolymerization
}

\author{
Qing-Hui Guo ${ }^{1}$, Manping $\mathrm{Jia}^{2}$, Zhichang Liu ${ }^{3}$, Yunyan Qiu ${ }^{1}$, Hongliang Chen ${ }^{1}$, \\ Dengke Shen ${ }^{1}$, Xuan Zhang ${ }^{1}$, Qing Tu ${ }^{4,5}$, Matthew R. Ryder ${ }^{6}$, Haoyuan Chen ${ }^{7}$, \\ Peng $\mathrm{Li}^{1}$, Yaobin $\mathrm{Xu}^{4,5}$, Penghao $\mathrm{Li}^{1}$, Zhijie Chen ${ }^{1}$, Gajendra S. Shekhawat ${ }^{4,5}$, \\ Vinayak P. Dravid ${ }^{4,5}$, Randall Q. Snurr ${ }^{7}$, Douglas Philp ${ }^{1}$, Andrew C.-H. Sue ${ }^{8}$, \\ Omar K. Farha ${ }^{1}$, Marco Rolandi ${ }^{2}$, J. Fraser Stoddart ${ }^{1,8,9 *}$
}

${ }^{1}$ Department of Chemistry, ${ }^{4}$ Department of Materials Science and Engineering, ${ }^{5}$ NUANCE Center, and ${ }^{7}$ Department of Chemical and Biological Engineering, Northwestern University, 2145 Sheridan Road, Evanston, Illinois 60208, USA.

${ }^{2}$ Department of Electrical Engineering, University of California Santa Cruz, Santa Cruz, California 95064, USA.

${ }^{3}$ School of Science, Westlake University, 18 Shilongshan Road, Hangzhou 310024, China. ${ }^{6}$ Neutron Scattering Division, Oak Ridge National Laboratory, Oak Ridge, TN 37831, USA.

${ }^{8}$ Institute for Molecular Design and Synthesis, Tianjin University, Tianjin 300072, China. ${ }^{9}$ School of Chemistry, University of New South Wales, Sydney, NSW 2052, Australia. *Corresponding author. Email: stoddart@northwestern.edu 


\section{Table of Contents}

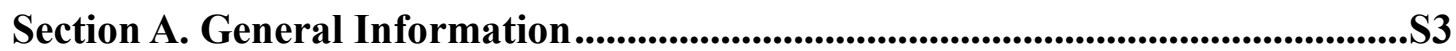

Section B. Synthesis of Monomer ...............................................................................6

Section C. X-Ray Crystallographic Characterization ...........................................S7

Section D. Topochemical Photopolymerization ..........................................................S12

1. In-Situ Single-Crystal X-Ray Diffractometer.........................................S12

2. Sunlight-Triggered Photopolymerization .....................................................S15

2. Gram-Scale Photopolymerization ..........................................................

3. In-Situ Powder X-Ray Diffractometer .......................................................S21

Section E. Scanning Electron Microscopy (SEM) ....................................................S23

Section F. Transmission Electron Microscopy (TEM) ............................................S24

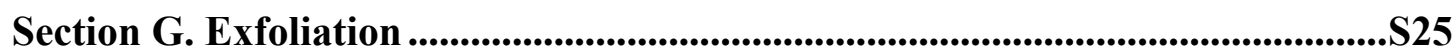

Section H. Nanoindentation .............................................................................S26

Section I. DFT Calculations and Response Properties ..........................................S28

Section J. Environmental and Chemical Stabilities .....................................................S31

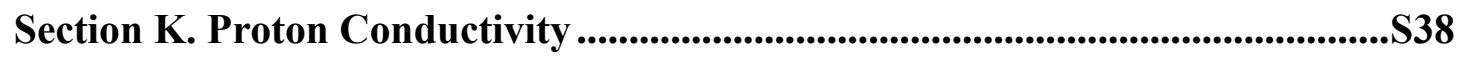

Section L. NMR Spectroscopy ...................................................................................S43

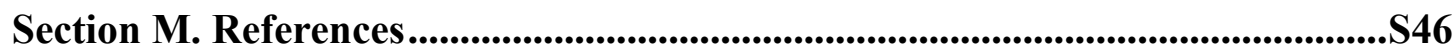




\section{Section A. General Information}

All commercially available reagents were used as received. Anhydrous MeCN was prepared by solvent drying system. Infrared spectra (IR) were recorded using a Nexus 870 spectrometer. Nuclear magnetic resonance (NMR) spectra were recorded on Bruker Avance 500 spectrometers, with working frequencies of $500 \mathrm{MHz}$ for ${ }^{1} \mathrm{H}$ and $125 \mathrm{MHz}$ for ${ }^{13} \mathrm{C}$ nuclei, respectively. Chemical shifts were reported in ppm relative to the signals corresponding to the residual non-deuterated solvents $\left(\mathrm{CD}_{3} \mathrm{CN}: \delta_{\mathrm{H}}=1.94\right.$ and $\delta_{\mathrm{C}}=118.3$ ppm). Abbreviations are used in the description of NMR data as follows: chemical shift $(\delta, \mathrm{ppm})$, multiplicity $(\mathrm{s}=$ singlet, $\mathrm{d}=$ doublet $)$, coupling constant $(J, \mathrm{~Hz})$. Highresolution mass spectra (ESI-HRMS) were measured on a Finnigan LCQ iontrap mass spectrometer. Single-crystal X-ray diffraction (SCXRD) data were collected on a Bruker APEX-II CCD diffractometer. Powder X-ray diffraction (PXRD) patterns were measured on an STOE-STADIMP powder diffractometer $\left(\mathrm{Cu}-\mathrm{K} \alpha_{1}\right.$ radiation, $\lambda=$ $1.54056 \AA$ ). Scanning electron microscopy (SEM) images were collected on a Hitachi SU8030 SEM. Transmission electron microscopy (TEM) images were performed on a JEOL ARM300F GrandARM. Thermogravimetric analyses (TGA) and differential scanning calorimetry (DSC) were performed on a TGA/DCS 1 system. Solid-state cross-polarization magic angle spinning (CPMAS) ${ }^{13} \mathrm{C}$ NMR spectroscopy was recorded on a $400 \mathrm{MHz}$ Bruker Avance III HD system. Atomic force microscopy (AFM) was performed on a SPID Bruker FastScan AFM. Nanoindentation was performed on a Hysitron 950 Tribolndenter. Chanzon High Power Led Chips (UV 365nm / 900mA / DC $9 \mathrm{~V}-11 \mathrm{~V} / 10 \mathrm{~W}$ ) were used for the irradiation experiments. Xenon Light Source 300W Monochromatic Light (MAX 350) with a $254 \mathrm{~nm}$ filter to study depolymerizations. 


\section{Methods}

Photopolymerization for in-situ single-crystal X-ray diffractometer: One single crystal of monomer $1 \cdot 3 \mathrm{BF}_{4}$ was selected, and a full set of diffraction data was collected in order to determine the structure. This crystal was irradiated-a $10 \mathrm{~W}, 365 \mathrm{~nm}$ LED, about $1.5 \mathrm{~cm}$ from the crystal-directly on the goniometer pin at $100 \mathrm{~K}$ for $3 \mathrm{~h}$ and another set of data collection afforded the structure of intermediate. Then, the same crystal was irradiated for another $6 \mathrm{~h}$ at $100 \mathrm{~K}$ in order to afford (Figure S3) the final polymer.

Gram-scale photopolymerizations: One gram of monomer crystals was obtained from a 1:10 mixture of $\mathrm{MeCN}$ and $i \operatorname{Pr}_{2} \mathrm{O}$. The freshly prepared crystals were suspended in the mother liquor and kept in a $30-\mathrm{mL}$ glass vial. The vial was placed closely under an LED. The crystals were irradiated with a 365-nm LED light for $9 \mathrm{~h}$. The vial was shaken gently every hour in order to make sure the crystals were being irradiated homogeneously. The whole procedure was carried out (Figure S10) in a fume hood. Polymerizations were monitored by ${ }^{1} \mathrm{H}$ NMR spectroscopy, IR and CPMAS ${ }^{13} \mathrm{C}$ NMR spectroscopies further identified the final products. The yield was calculated using the equation, $\mathrm{Y}=\mathrm{Mp} / \mathrm{Mm} \times 100 \%$, where $\mathrm{Y}$ is the yield, $\mathrm{Mp}$ is the weight of the singlecrystalline polymer, and $\mathrm{Mm}$ is the weight of the monomer.

Computational electronic and mechanical properties: Density functional theory (DFT) was used to calculate the electronic properties of the polymer using the B3LYPD3 functional and all-electron basis sets similar to our previous work on supramolecular and framework materials ${ }^{49,25}$. The electronic band gap of the ionic polymer crystal is predicted to be $3.72 \mathrm{eV}$, and the values of the anisotropic dielectric constants and refractive indices are reported in the SI. The anisotropic Young's moduli of the polymer were computed using a composite method (HF-3C), and the 3D representation is provided in the SI.

\section{Proton conductivities:}

Crystalline polymers were crushed into powders and drop-cast (Figure S32) on the twoterminal devices. Alternating current (a.c.) potentials were applied to the polymer hydrated at different relative humidities. The Nyquist plots were matched with a simple equivalent. The Constant Phase Element (CPE) was used to describe the non-ideal capacitive effect of the interface between the material and contacts. We also measured (Figure S36) the proton conductivity of Nafion using the same device: it was $\sim 0.1 \mathrm{~S}$ $\mathrm{cm}^{-1}$. 

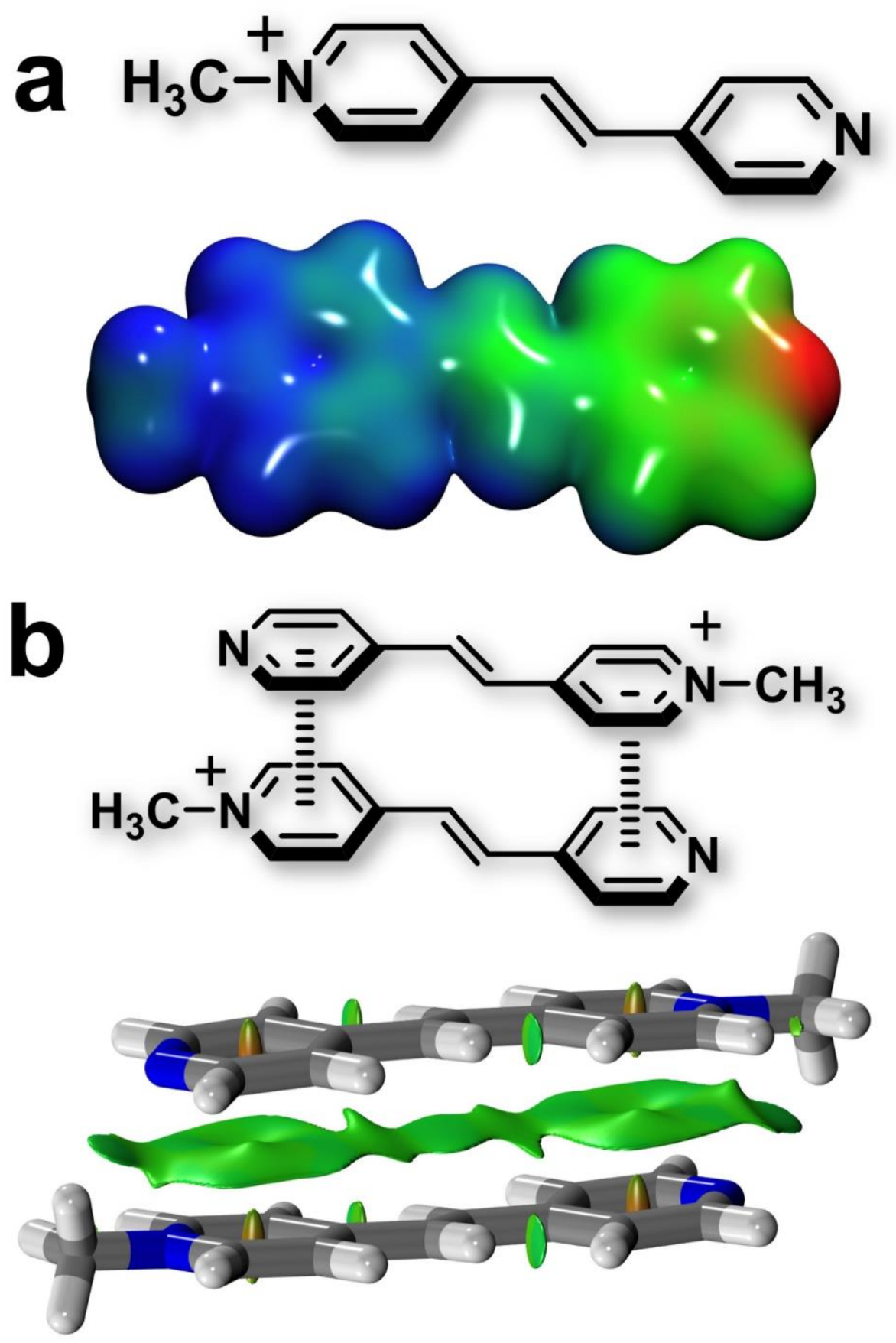

Figure S1. a, Electrostatic potential (E)-1-methyl-4-(2-(pyridin-4-yl)vinyl)pyridin-1ium) mapped to the total electron density surface. $\operatorname{Red}=$ negative; blue = positive. $\mathbf{b}$, Reduced density gradient analysis of the non-covalent interactions in the (E)-1-methyl4-(2-(pyridin-4-yl)vinyl)pyridin-1-ium) dimer calculated using NCIPLOT $3.0^{1}$. All the electronic structure calculations were run using Gaussian 16 Rev. B01 ${ }^{2}$. The calculations we performed using the MN15 functional ${ }^{3}$ and the def2-TZVP basis set ${ }^{4}$. 

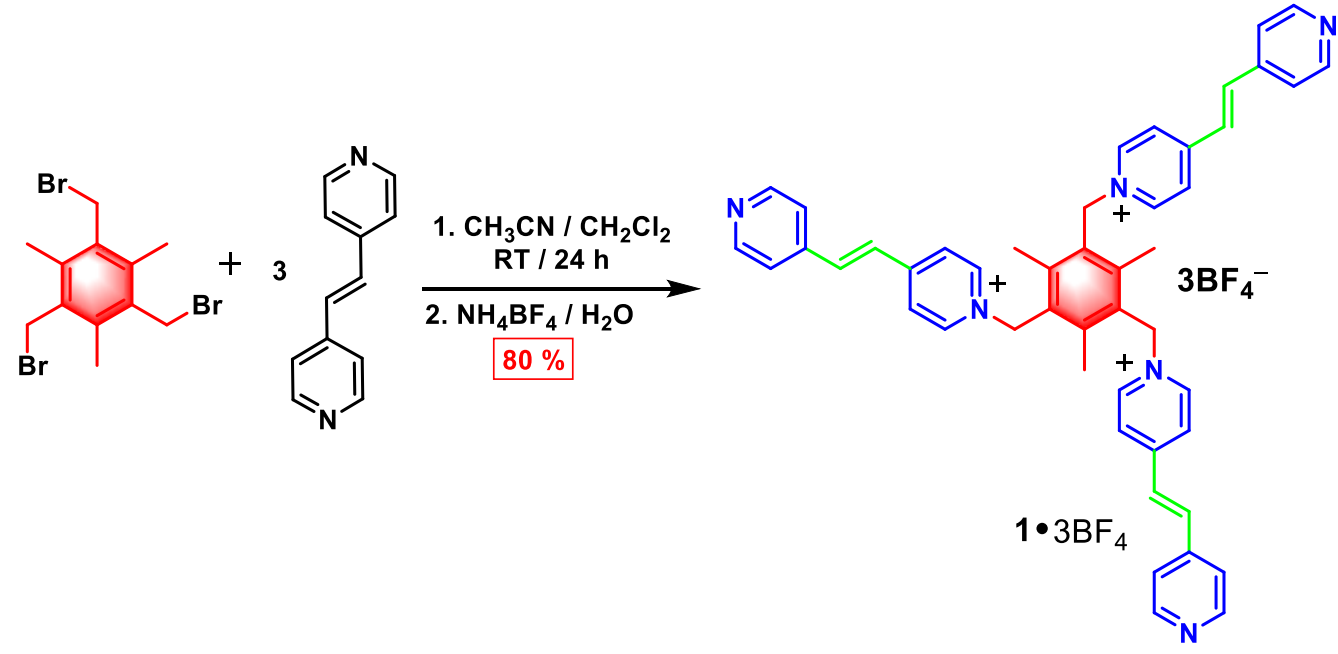

Scheme S1. Synthesis of $\mathbf{1} \cdot 3 \mathrm{BF}_{4}$

1•3BF4: A solution of 1,3,5-tris(bromomethyl)-2,4,6-trimethylbenzene (800 mg, 3 mmol) in dry $\mathrm{CH}_{2} \mathrm{Cl}_{2}(20 \mathrm{~mL})$ and $\mathrm{MeCN}(5 \mathrm{~mL})$ was added dropwise during $12 \mathrm{~h}$ by syringe to a solution of (E)-1,2-di(pyridin-4-yl)ethene $(8.20 \mathrm{~g}, 45 \mathrm{mmol})$ in anhydrous $\mathrm{CH}_{2} \mathrm{Cl}_{2}(30 \mathrm{~mL})$ and $\mathrm{MeCN}(60 \mathrm{~mL})$ under an $\mathrm{N}_{2}$ atmosphere. The flask was wrapped with aluminium foil and the mixture was stirred continuously for $24 \mathrm{~h}$ at room temperature. The resulting white precipitate was collected by filtration, washed with $\mathrm{CH}_{2} \mathrm{Cl}_{2}(3 \times 50 \mathrm{~mL})$ and $\mathrm{MeCN}(3 \times 50 \mathrm{~mL})$, and dried. The precipitate was dissolved in $\mathrm{H}_{2} \mathrm{O}(1.5 \mathrm{~L})$. A solution of $\mathrm{NH}_{4} \mathrm{BF}_{4}(6.0 \mathrm{~g})$ in $\mathrm{H}_{2} \mathrm{O}(20 \mathrm{~mL})$ was added to the abovementioned solution of the monomer. The resulting white precipitate was collected by filtration, washed with $\mathrm{H}_{2} \mathrm{O}(3 \times 50 \mathrm{~mL})$, and dried. The remaining solid was recrystallized from $\mathrm{MeCN}$ and $i \mathrm{Pr}_{2} \mathrm{O}$ to afford $1 \cdot 3 \mathrm{BF}_{4}$ as a colorless crystalline solid $(2.32 \mathrm{~g}, 2.4 \mathrm{mmol})$ in $80 \%$ yield. ${ }^{1} \mathrm{H} \mathrm{NMR}\left(500 \mathrm{MHz}, \mathrm{CD}_{3} \mathrm{CN}, 298 \mathrm{~K}\right) \delta_{H}=8.68(\mathrm{~d}, J=$ $6.1 \mathrm{~Hz}, 12 \mathrm{H}), 8.51(\mathrm{~d}, J=6.9 \mathrm{~Hz}, 12 \mathrm{H}), 8.13(\mathrm{~d}, J=7.0 \mathrm{~Hz}, 12 \mathrm{H}), 7.76(\mathrm{~d}, J=16.5 \mathrm{~Hz}$, 12H), 7.62-7.55 (m, 18H), 5.87 (s, 12H), 2.27 (s, 18H); ${ }^{13} \mathrm{C} \mathrm{NMR} \mathrm{(125} \mathrm{MHz,} \mathrm{CD}_{3} \mathrm{CN}$, 298 K) $\delta_{C}=17.2,58.9,122.3,125.9,127.6,129.3,139.4,142.6,144.0,144.8,151.3$, 153.9; ESI-HRMS Calcd for $\mathrm{C}_{48} \mathrm{H}_{45} \mathrm{~F}_{12} \mathrm{~B}_{3} \mathrm{~N}_{6}: m / z=879.3759\left[M-\mathrm{BF}_{4}\right]^{+}, 396.1862[M$ $\left.-2 \mathrm{BF}_{4}\right]^{2+}$; found: $879.3772\left[M-\mathrm{BF}_{4}\right]^{+}, 396.1874\left[M-2 \mathrm{BF}_{4}\right]^{2+}$. 


\section{Section C. X-Ray Crystallographic Characterization}

Single crystals of $\mathbf{1} \cdot 3 \mathrm{BF}_{4}$ were obtained after only one day by slow vapor diffusion of $i \mathrm{Pr}_{2} \mathrm{O}$ into a MeCN solution. In order to monitor the polymerization by in-situ singlecrystal X-ray diffractometer, one single crystal was selected and mounted in inert oil and transferred to the cold $\mathrm{N}_{2}$ gas stream of a Bruker Kappa APEX CCD area detector diffractometer. The crystal was kept at $100 \mathrm{~K}$ during the data collection. After a set of data collection was finished, the same crystal was irradiated directly on the diffractometer under $100 \mathrm{~K}$ for $3 \mathrm{~h}$ to afford the intermediate crystal. This crystal was kept at $100 \mathrm{~K}$ during the data collection. After this set of data collection was finished, the same crystal was irradiated for another $6 \mathrm{~h}$ under $100 \mathrm{~K}$ to afford the final polymeric crystal. This crystal was kept at $100 \mathrm{~K}$ for the data collection. Even though, as the polymerization proceeded, the quality of the single crystal decreased. Both the singlecrystal structures of the intermediate and the polymer, however, could be solved. Considering that we are dealing with a challenging single-crystal-to-single-crystal polymerization, it should be acceptable that the quality of polymer crystal cannot compete with the standard compounds, which are obtained by well-known procedures. Using Olex $2^{5}$, the above data were resolved with the ShelXT ${ }^{6}$ structure solution program and all the structures (the monomer, the intermediate and the final polymer) were refined with the ShelXL ${ }^{7}$ package using least-squares minimization.

All crystallographic data for the structures reported here have been deposited on to the Cambridge Crystallographic Data Centre (CCDC) and can be downloaded free of charge from www.ccdc.cam.ac.uk/data request/cif. The crystallographic information, 
structural parameters for $\mathbf{1} \bullet 3 \mathrm{BF}_{4}$ monomer, intermediate crystal, and $\mathbf{2} \bullet \mathrm{nPF}_{6}$ polymer are as follows.

1•3BF $\mathrm{BF}_{4}$ Monomer (CCDC number 1952786) Crystal Data for $\mathrm{C}_{48} \mathrm{H}_{47.67} \mathrm{~B}_{3} \mathrm{~F}_{12} \mathrm{~N}_{6}$ $\mathrm{O}_{1.33}(M=990.35 \mathrm{~g} / \mathrm{mol})$ : monoclinic, space group $C 12 / c 1$ (no. 15$), a=58.143(2), b=$ 11.9245(5), $c=15.4738(7) \AA, \alpha=90, \beta=102.308(3), \gamma=90^{\circ}, V=10481.8(8) \AA^{3}, Z=$ $8, T=100.02 \mathrm{~K}, \mu(\mathrm{Cu} \mathrm{K} \alpha)=0.908 \mathrm{~mm}^{-1}$, Dcalc $=1.255 \mathrm{~g} / \mathrm{cm}^{3}$. The final $R_{1}$ was $0.1079(I>2 \sigma(I))$ and $w R_{2}$ was 0.3034 (all data).

Intermediate Crystal (CCDC number 1952787) Crystal Data for $\mathrm{C}_{96} \mathrm{H}_{90} \mathrm{~B}_{6} \mathrm{~F}_{24} \mathrm{~N}_{12}$ $\mathrm{O}_{3}(M=1980.65 \mathrm{~g} / \mathrm{mol})$ : monoclinic, space group $C 12 / c 1$ (no. 15$), a=90, \beta=$ 103.731(2), $\gamma=90^{\circ}, V=10526.6(5) \AA^{3}, Z=4, T=100 \mathrm{~K}, \mu(\mathrm{Cu} \mathrm{K \alpha})=0.907 \mathrm{~mm}^{-1}$, Dcalc $=1.250 \mathrm{~g} / \mathrm{cm}^{3}$. The final $R_{1}$ was $0.1469(I>2 \sigma(I))$ and $w R_{2}$ was 0.4541 (all data). CheckCIF alert justifications

THETM01_ALERT_3_B The value of sine(theta_max)/wavelength is less than 0.575 Calculated sin(theta_max $) /$ wavelength $=0.5748$

Author Response: The single crystals after light irradiation did not diffract well and a resolution of $0.87 \AA$ was obtained after multiple trials.

PLAT084_ALERT_3_B High wR2 Value (i.e. > 0.25) 0.45 Report

Author Response: This outcome is a result of the less-than-ideal diffraction data quality of single crystals after light irradiation.

PLAT306_ALERT_2_B Isolated Oxygen Atom (H-atoms Missing?) ....... O1 Check Author Response: In view of the less-than-ideal diffraction data quality of single crystals after light irradiation, hydrogen atoms are not added to solvent $\mathrm{H}_{2} \mathrm{O}$ molecules. 
PLAT340_ALERT_3_B Low Bond Precision on C-C Bonds $0.01223 \AA$.

Author Response: This outcome is the result of the less-than-ideal diffraction data quality of single crystals after light irradiation.

2॰nBF 4 Polymer (CCDC number 1952788) Crystal Data for $\mathrm{C}_{48} \mathrm{H}_{45} \mathrm{~B}_{3} \mathrm{~F}_{12} \mathrm{~N}_{6} \mathrm{O}_{1} \quad(M=$ $982.33 \mathrm{~g} / \mathrm{mol}$ ): monoclinic, space group $C 12 / c 1$ (no. 15$), a=57.282(7), b=$ 11.7334(15), $\quad c=16.2714(18) \AA, \quad \alpha=90, \quad \beta=104.674(7), \quad \gamma=90^{\circ}, \quad V=$ 10526.6(5) $\AA^{3}, Z=8, T=102(2) \mathrm{K}, \mu(\mathrm{Cu} \mathrm{K \alpha})=0.891 \mathrm{~mm}^{-1}$, Dcalc $=1.233 \mathrm{~g} / \mathrm{cm}^{3}$. The final $R_{1}$ was $0.1698(I>2 \sigma(I))$ and $w R_{2}$ was 0.4877 (all data).

CheckCIF alert justifications

THETM01_ALERT_3_A The value of sine(theta_max)/wavelength is less than 0.550 Calculated $\sin ($ theta_max $) /$ wavelength $=0.4348$

Author Response: The in situ polymerization of the single crystal of the monomers resulted in poor diffraction of the single crystal of the polymer product. The best resolution obtained is $1.15 \AA$.

PLAT084_ALERT_3_A High $w R_{2}$ Value (i.e. > 0.25) 0.49 Report

Author Response: This high $w R_{2}$ value is attributed to the poor diffraction of the product single crystal after in situ polymerization of the monomer single crystal.

PLAT088_ALERT_3_A Poor Data / Parameter Ratio 5.76 Note

Author Response: This poor data / parameter ratio is attributed to the poor diffraction of the product single crystal after in situ polymerization of the monomer single crystal. A number of restraints were applied during the refinement. 

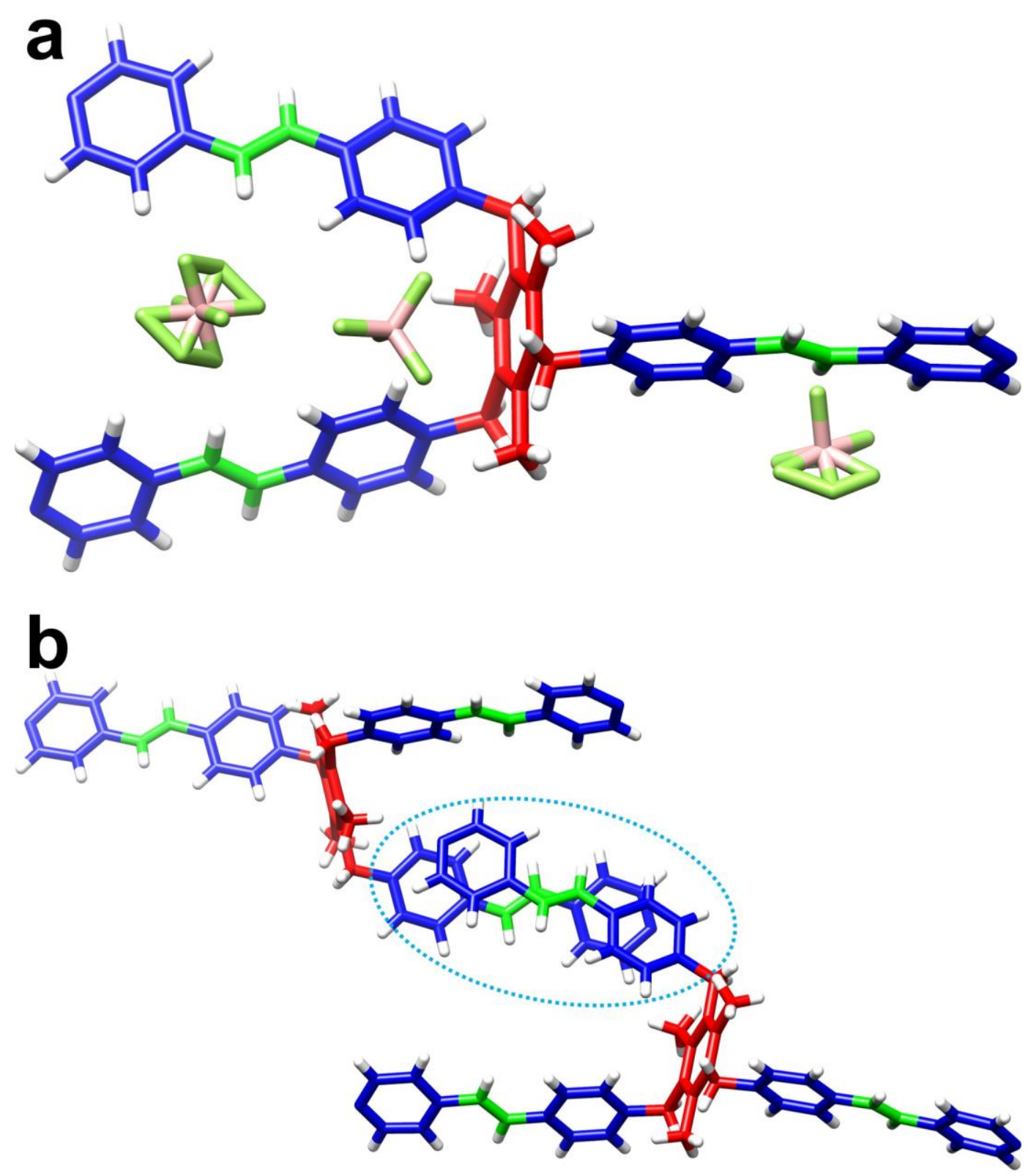

Figure S2. The three $\mathrm{BF}_{4}^{-}$counterions and the packing mode of the third arm. a, Two $\mathrm{BF}_{4}^{-}$anions are disordered in the crystal. $\mathbf{b}$, The third arms (labeled by the blue circle) cannot form self-complementary interactions with the arms from adjacent molecules. 
Table S1. Parameters of single crystals of monomer, intermediate and polymer.

\begin{tabular}{|c|c|c|c|c|c|c|c|c|c|}
\hline & Space Group & $\alpha\left(^{\circ}\right)$ & $\beta\left(^{\circ}\right)$ & $\gamma\left({ }^{\circ}\right)$ & $a(\AA)$ & $b(\AA)$ & $c(\AA)$ & $V\left(\AA^{3}\right)$ & $\begin{array}{l}\text { Change } \\
\text { of } V / \%\end{array}$ \\
\hline Monomer & $C 2 / c$ & 90 & 102.31 & 90 & 58.14 & 11.92 & 15.47 & 10481.8 & -- \\
\hline Intermediate & $C 2 / c$ & 90 & 103.73 & 90 & 57.90 & 11.88 & 15.75 & 10526.6 & +0.43 \\
\hline Polymer & $C 2 / c$ & 90 & 104.67 & 90 & 57.28 & 11.73 & 16.27 & 10579.5 & +0.93 \\
\hline
\end{tabular}




\section{Section D. Topochemical Photopolymerization}

\section{In-Situ Single-Crystal X-Ray Diffractometer}
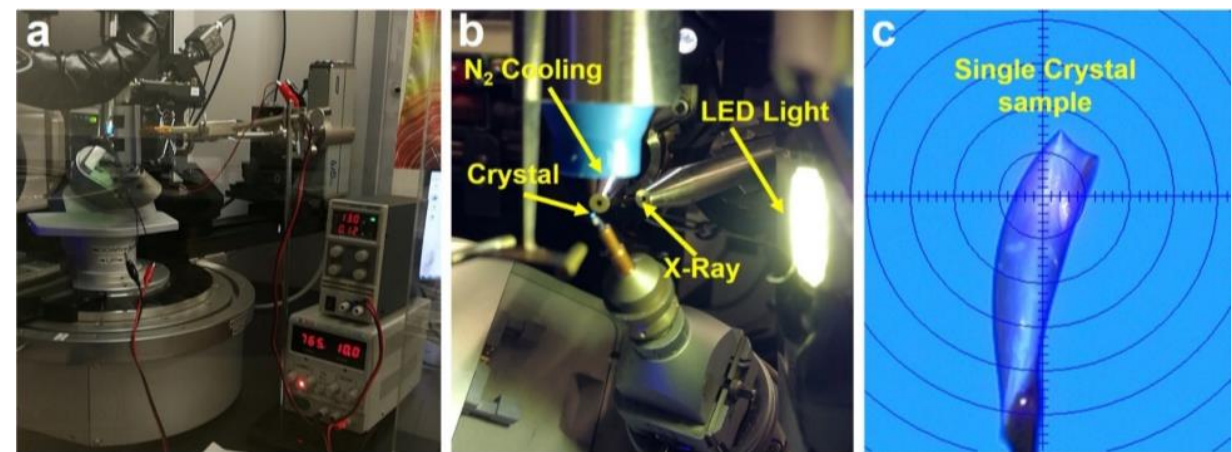

Figure S3. The setup for monitoring the photopolymerization by in-situ single-crystal X-ray diffractometry. a, Overview picture and $\mathbf{b}$, close up picture of the setup. c, A single crystal irradiated by a $10 \mathrm{~W}$ UV LED under $100 \mathrm{~K}$.
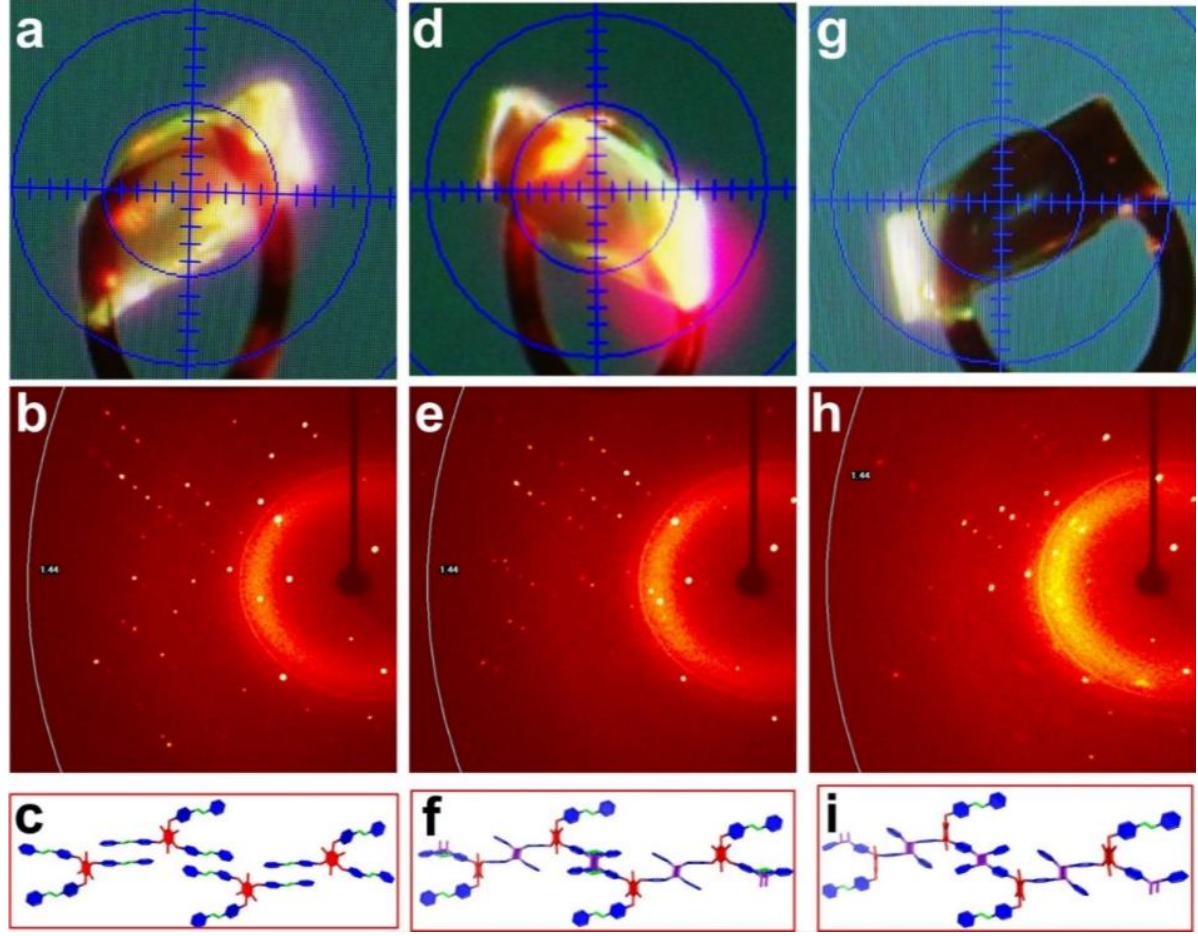

Figure S4. Overview of the single-crystal-to-single-crystal photopolymerization of one

single crystal. a, Monomer single crystal. b, Single frame of the monomer matrix. c, Single crystal (super)structure of monomer. d, Intermediate single crystal. e, Single frame of the intermediate matrix. f, Single-crystal structure of intermediate. $\mathbf{g}$, Polymer single crystal. h, Single frame of the polymer matrix. i, Single-crystal structure polymer. All the frames were recorded at $10 \mathrm{~s}$ exposure time. 


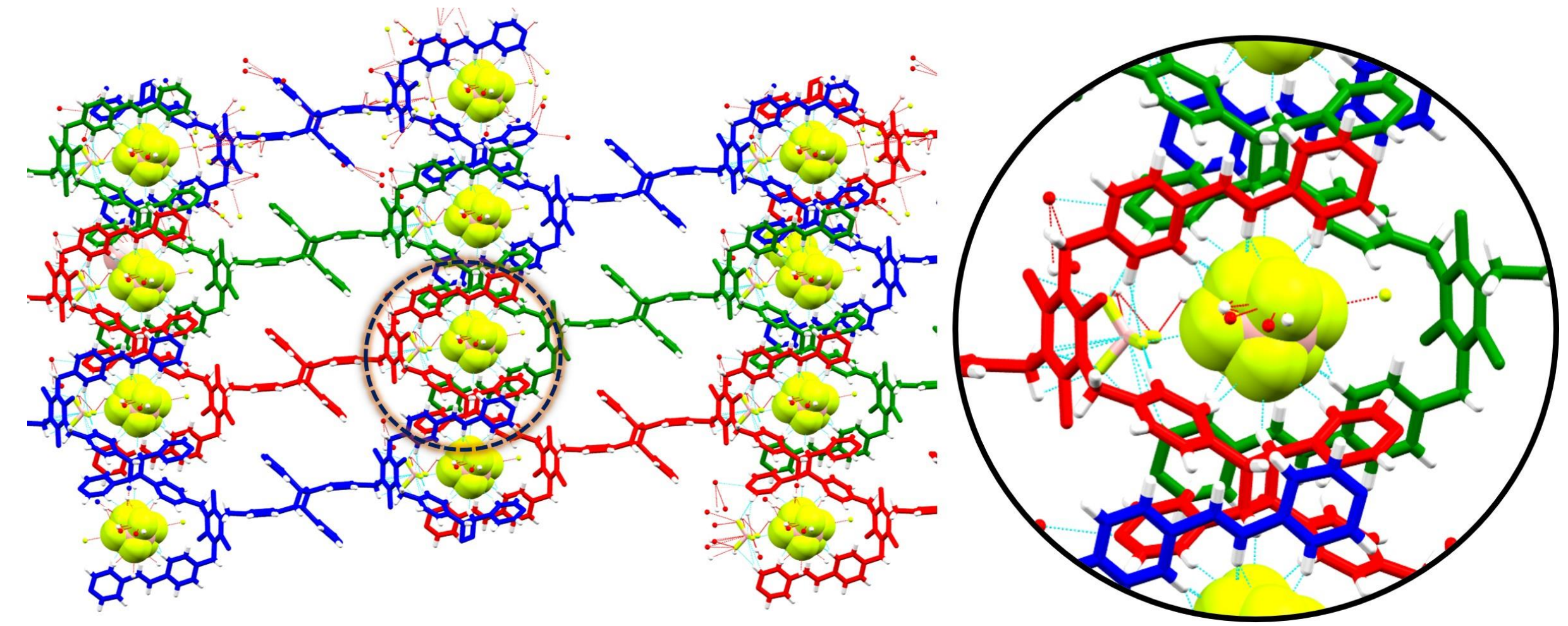

Figure S5. The multiple electrostatic interactions between $\mathrm{BF}_{4}^{-}$counterions and positively charged polymer chains in the monolayer. The zoomin picture emphasizes that one $\mathrm{BF}_{4}^{-}$counterion has multiple electrostatic interactions with six adjacent polymer chains. The adjacent chains are illustrated as stick representations, while the $\mathrm{BF}_{4}^{-}$counterions are disordered and shown as space-filling representations. 


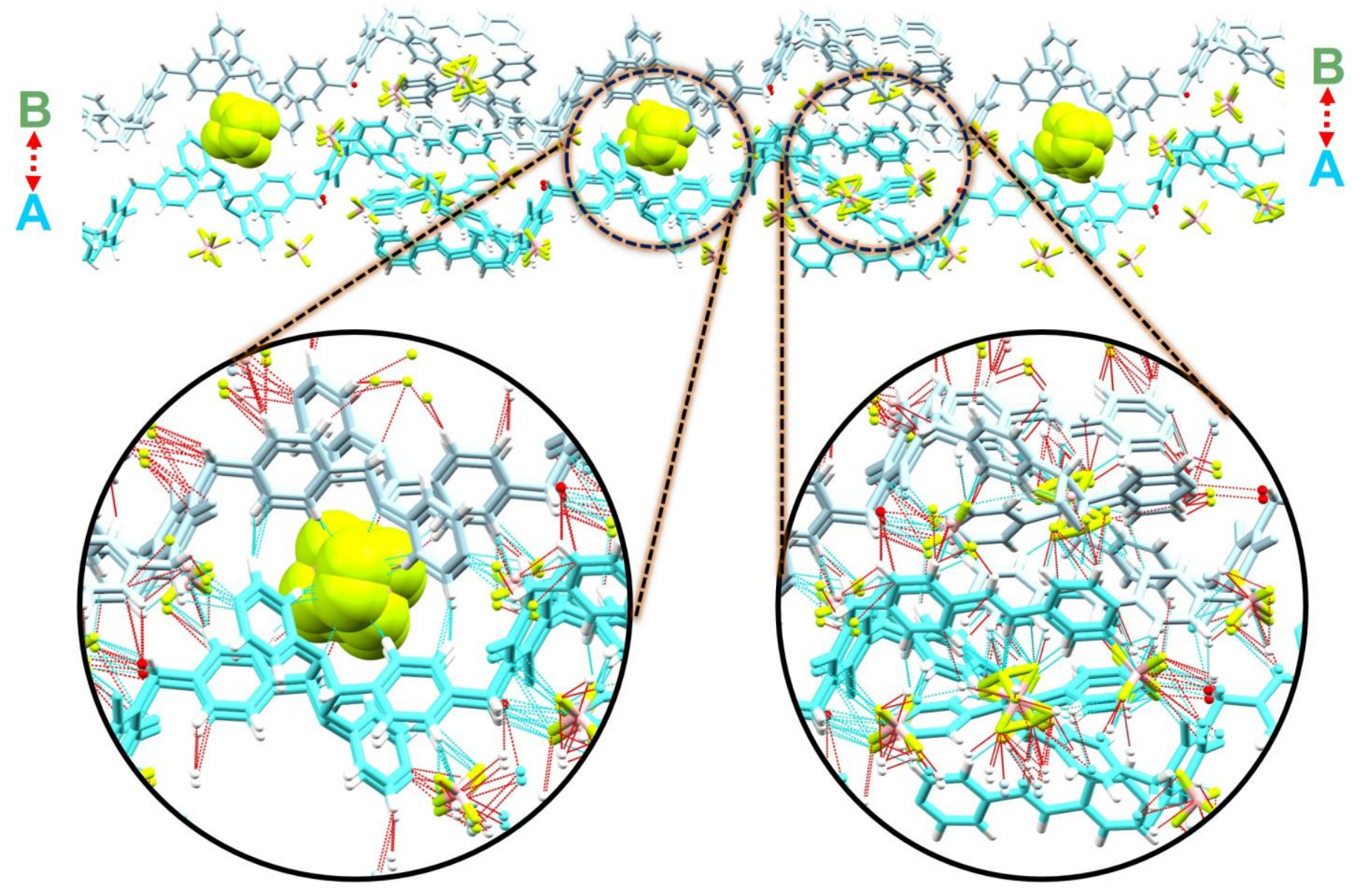

Figure S6. The electrostatic interactions and Van der Waals forces between two adjacent monolayers. The polymer chains are illustrated as stick representations, while the disordered $\mathrm{BF}_{4}^{-}$counterions which interact with two layers are shown as space-filling representations. 


\section{Sunlight-Triggered Photopolymerization}

Freshly prepared crystals were suspended in their mother liquor and kept in a 3-mL quartz cuvette. The cuvette was sealed and placed on the garden under the sunlight at Northwestern University, Evanston. The crystals were irradiated from 10 am to $4 \mathrm{pm}$ on July 23 and 24, 2019 (6 hours per day). The cuvette was shaken gently every hour in order to make sure the crystals were irradiated homogeneously. Four 6-mg aliquots of crystals were taken out at different time intervals $(1,3,6$, and $9 \mathrm{~h})$ and dissolved in $\mathrm{CD}_{3} \mathrm{CN}$ for ${ }^{1} \mathrm{H}$ NMR spectroscopy and PXRD. The final product was also identified by ${ }^{1} \mathrm{H}$ NMR spectroscopy and PXRD.
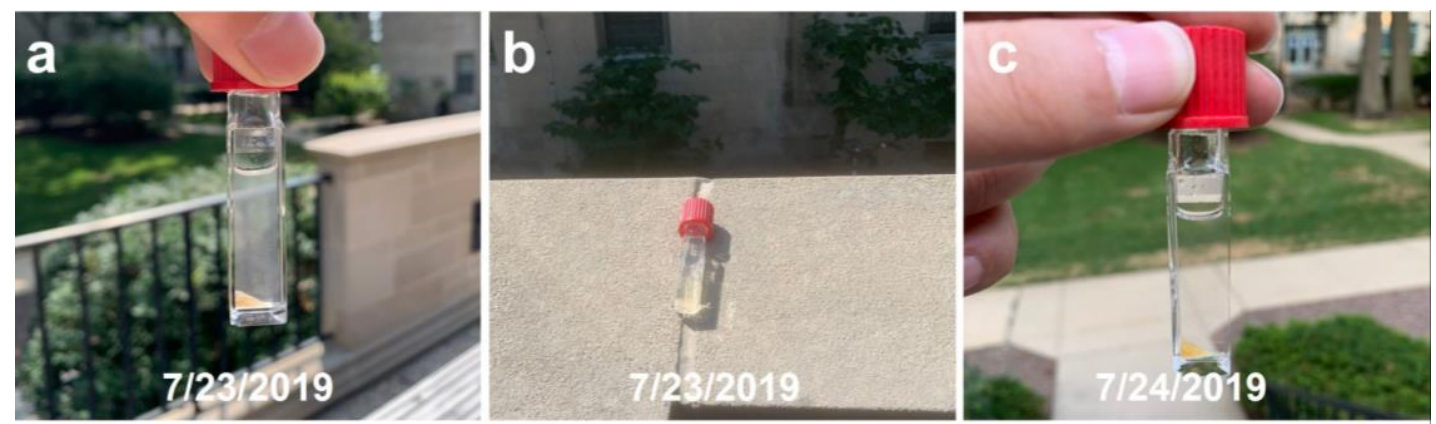

d $7 / 23 / 2019$

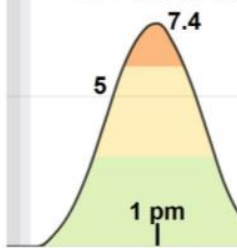

UV Index

\section{$7 / 24 / 2019$}

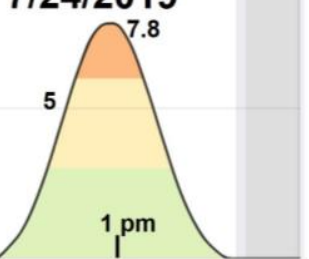

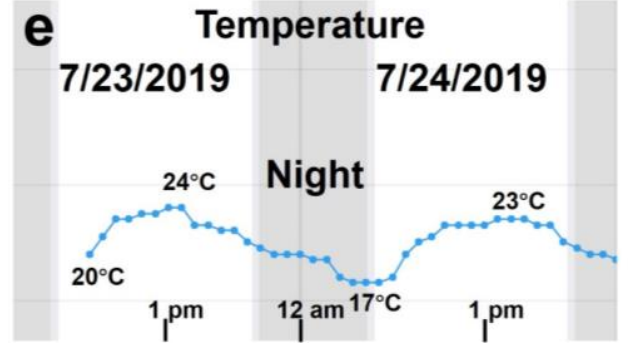

Figure S7. Photopolymerization was triggered by sunlight. Samples before (a), during (b) and after (c) irradiated by sunlight for $10 \mathrm{~h}$. The color of the samples changed from pale yellow to yellow. The UV index (d) and temperature trends (e) of the day on July 23 and 24, 2019. The UV index changed from 4.0 to 7.8 (10 am to $4 \mathrm{pm}$ ) and the temperature was about $296 \mathrm{~K}$ during the polymerization. The weather information was from Data Sources of WillyWeather (https://www.willyweather.com/). 

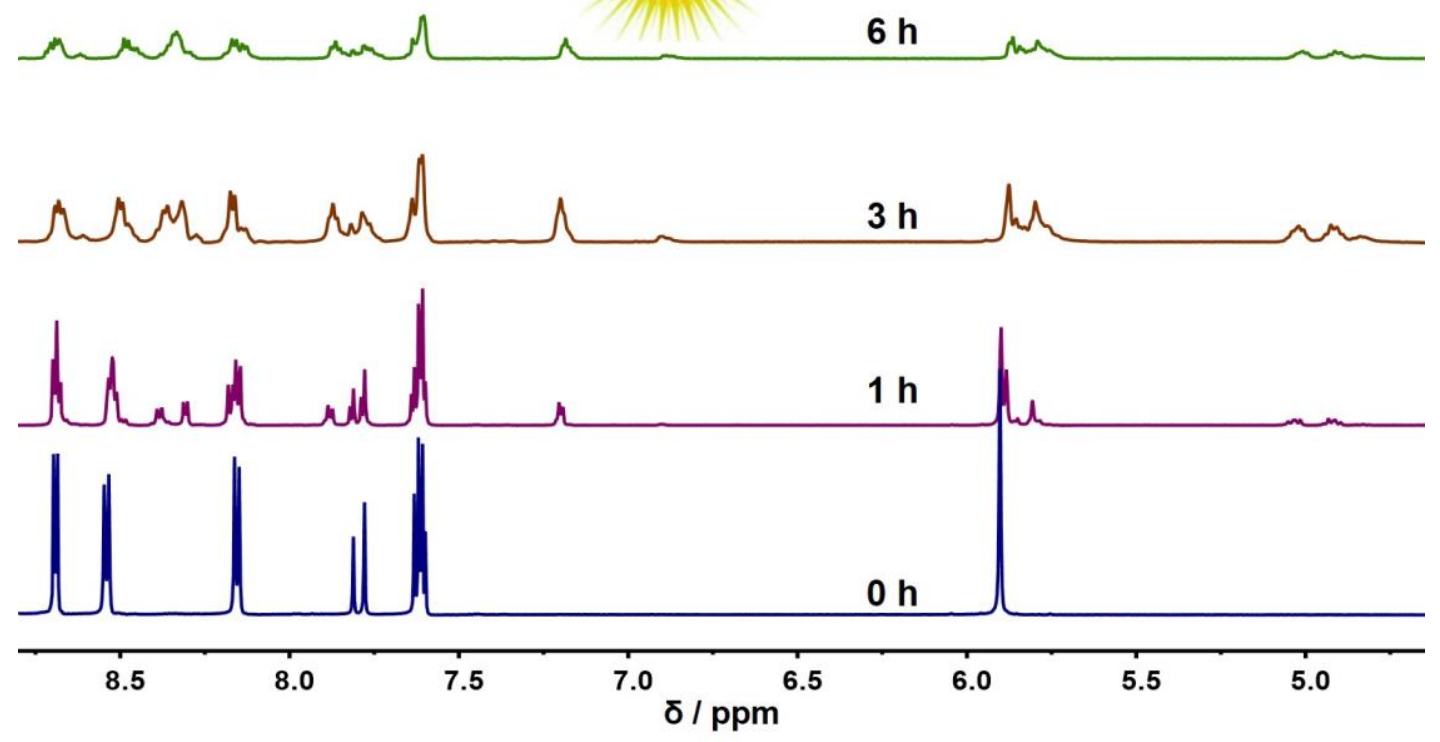

Figure S8. ${ }^{1} \mathrm{H}$ NMR Spectra for monitoring the polymerization. The samples were virtually insoluble in $\mathrm{CD}_{3} \mathrm{CN}$ after irradiation of $9 \mathrm{~h}$ by sunlight.

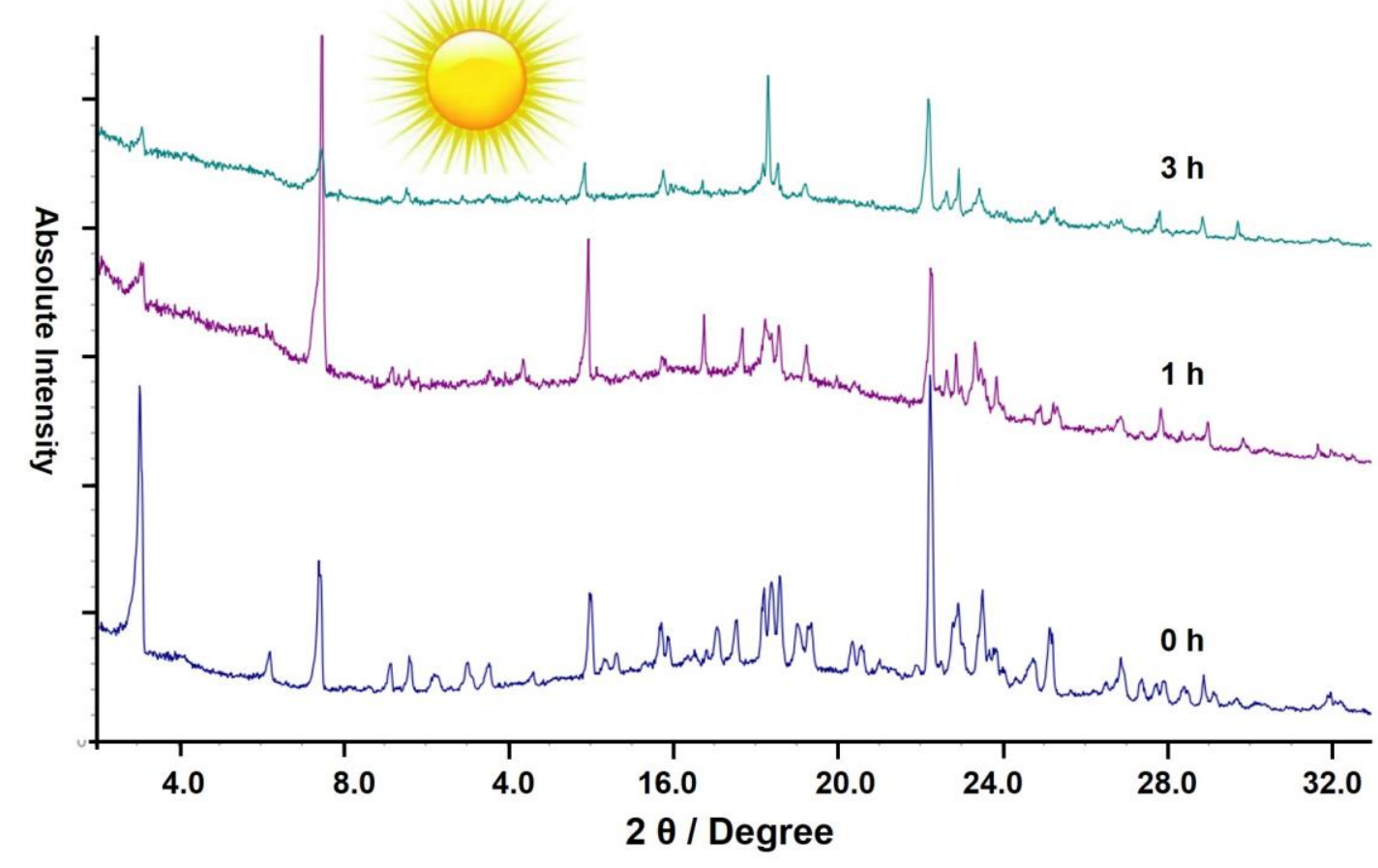

Figure S9. PXRD of the photopolymerization under sunlight at room temperature. 


\section{Gram-Scale Photopolymerization}

One gram of monomer crystals were grown in the mixture of $\mathrm{MeCN}$ and $i \operatorname{Pr}_{2} \mathrm{O}(1: 10)$.

The freshly prepared crystals were suspended in their mother liquor and kept in a 30$\mathrm{mL}$ glass vial. The vial was placed closely under the LED, which was connected with a cooling fan. The crystals were irradiated with $365 \mathrm{~nm}$ light for $9 \mathrm{~h}$ at room temperature. The vial was shaken gently every hour in order to make sure the crystals were irradiated homogeneously. Seven 3-mg aliquots of crystals were taken out at different time intervals $(0.25,0.5,1,3,6$, and $9 \mathrm{~h})$ and dissolved in $\mathrm{CD}_{3} \mathrm{CN}$ for recording ${ }^{1} \mathrm{H}$ NMR spectra. The final product was also identified by CPMAS ${ }^{13} \mathrm{C}$ NMR spectroscopy and IR spectrophotometry.
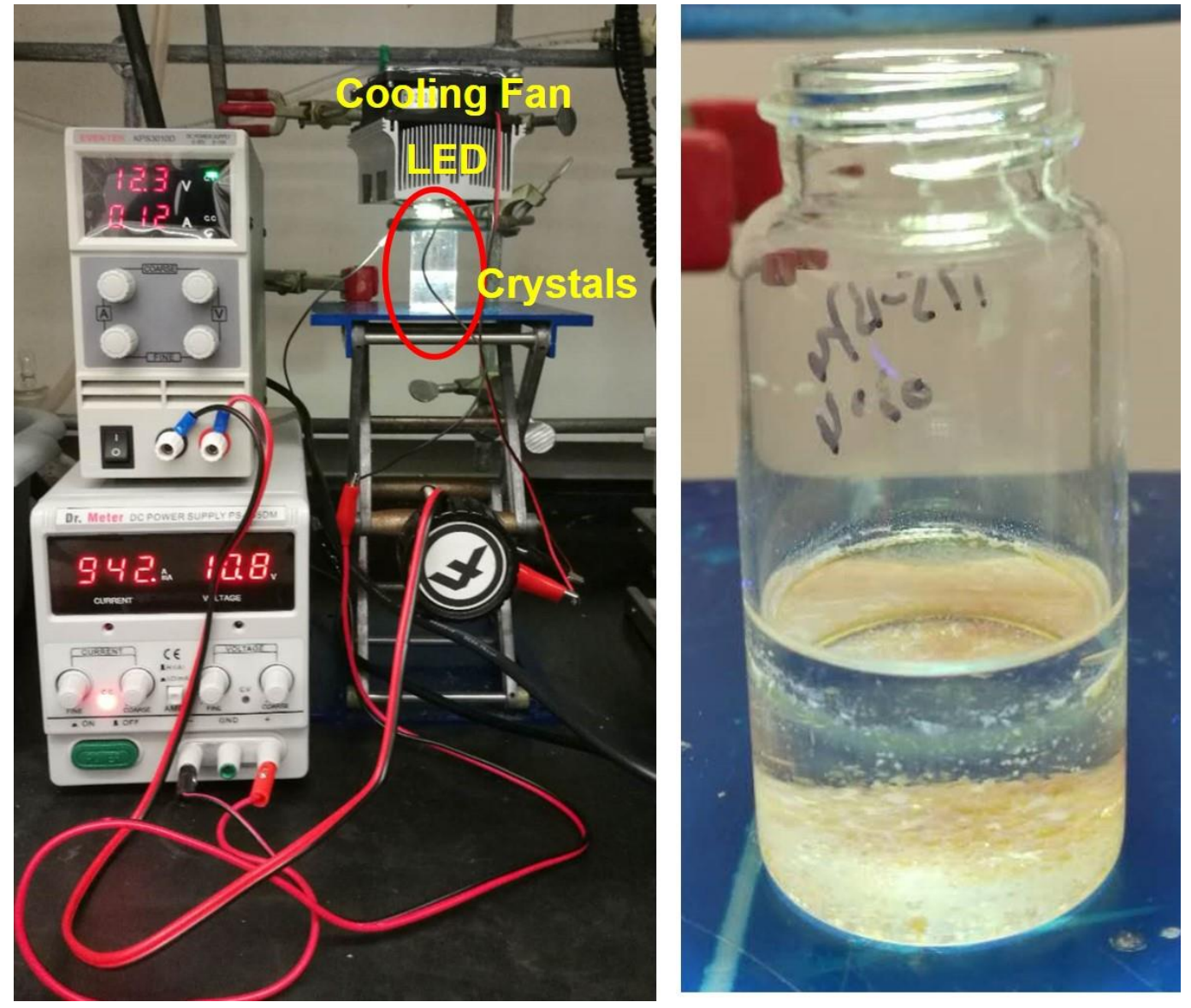

Figure S10. Custom-built setup in the hood 

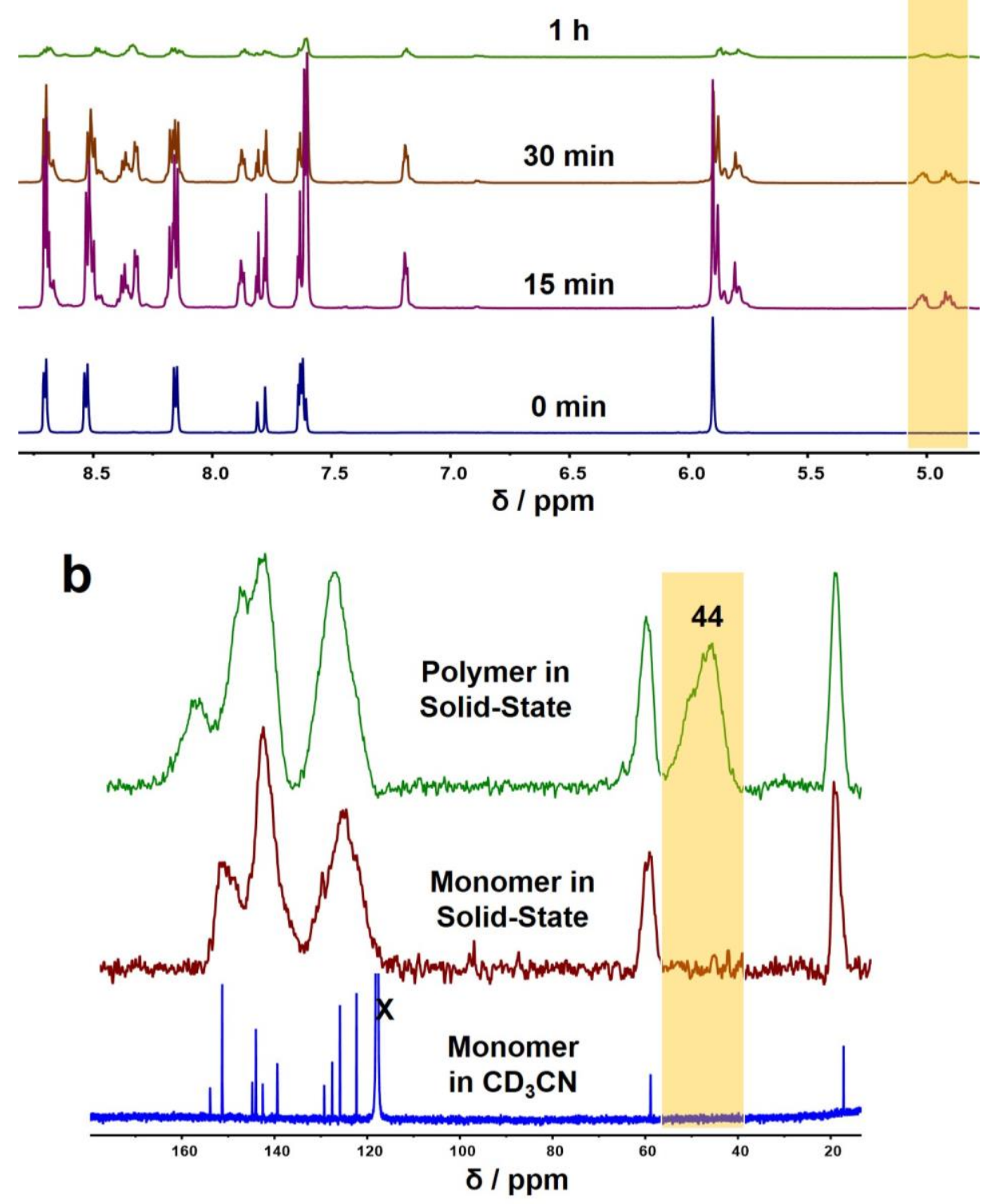

Figure S11. ${ }^{1} \mathrm{H}$ NMR and CPMAS ${ }^{13} \mathrm{C}$ NMR spectroscopies . a, The peaks around 5 ppm were assigned to protons in the newly formed cyclobutane rings. The polymeric crystals were virtually insoluble in $\mathrm{CD}_{3} \mathrm{CN}$ after irradiation of $3 \mathrm{~h}$. bo , The insoluble polymer was characterized by solid-state ${ }^{13} \mathrm{C}$ NMR spectroscopy. The new signal at $\delta$ $=44 \mathrm{ppm}$ showed the conversion of double bonds in monomer to cyclobutane rings in the polymer. Some third arms with double bonds still remain in the final structure of the polymer. The signal at $\delta \approx 60$ ppm belongs to these unreacted arms. 


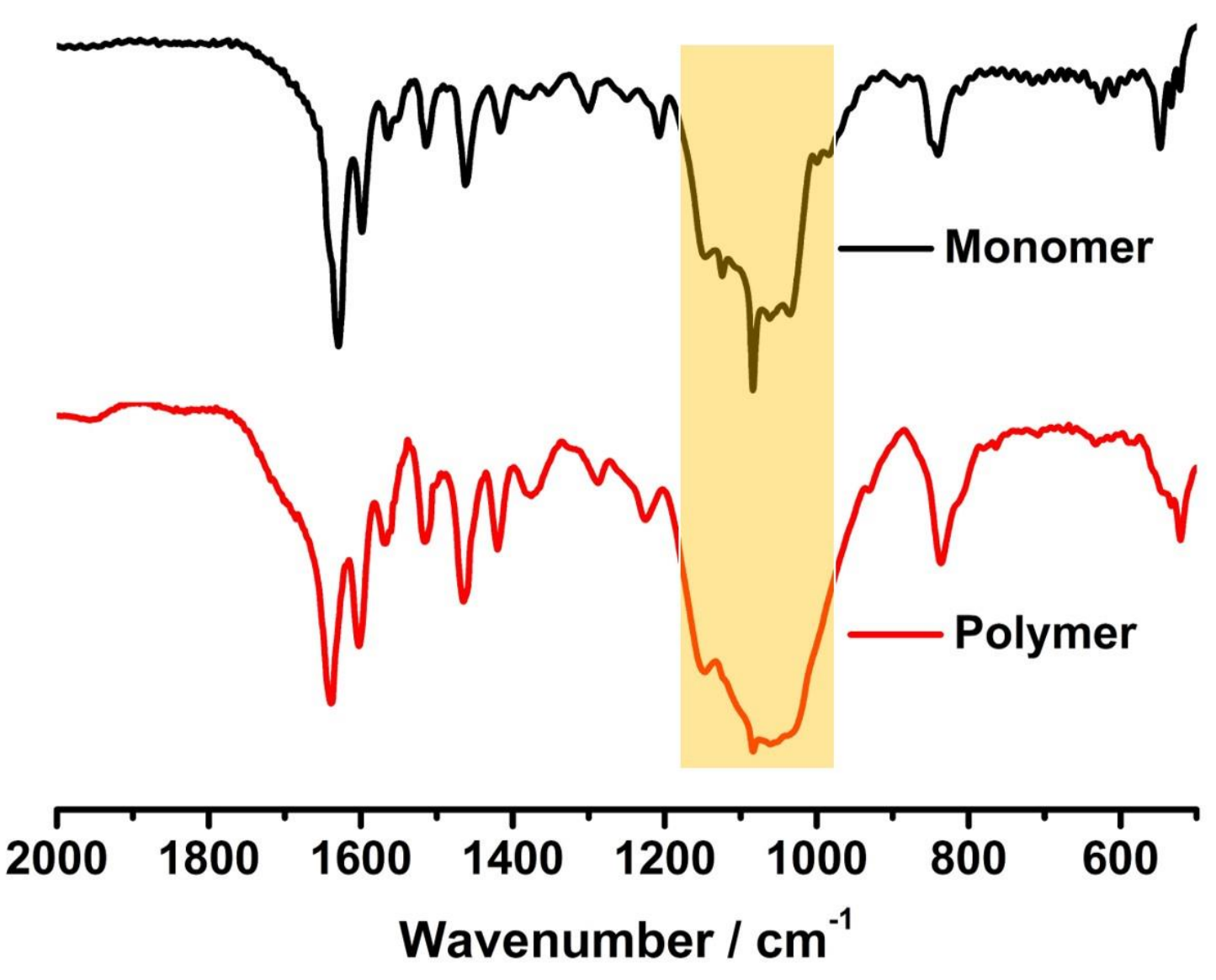

Figure S12. IR Spectra of monomer and polymer crystals. There are no other obvious changes in the IR spectra apart from the peaks in the range of 1020 to $1160 \mathrm{~cm}^{-1}$ became broad. The vibrational bands arising from newly formed bonds in cyclobutane rings were not present in the IR spectra. The peaks around the $1600 \mathrm{~cm}^{-1}$ belonging to the double bonds did not disappear because there is still one arm left in the polymer structure. 


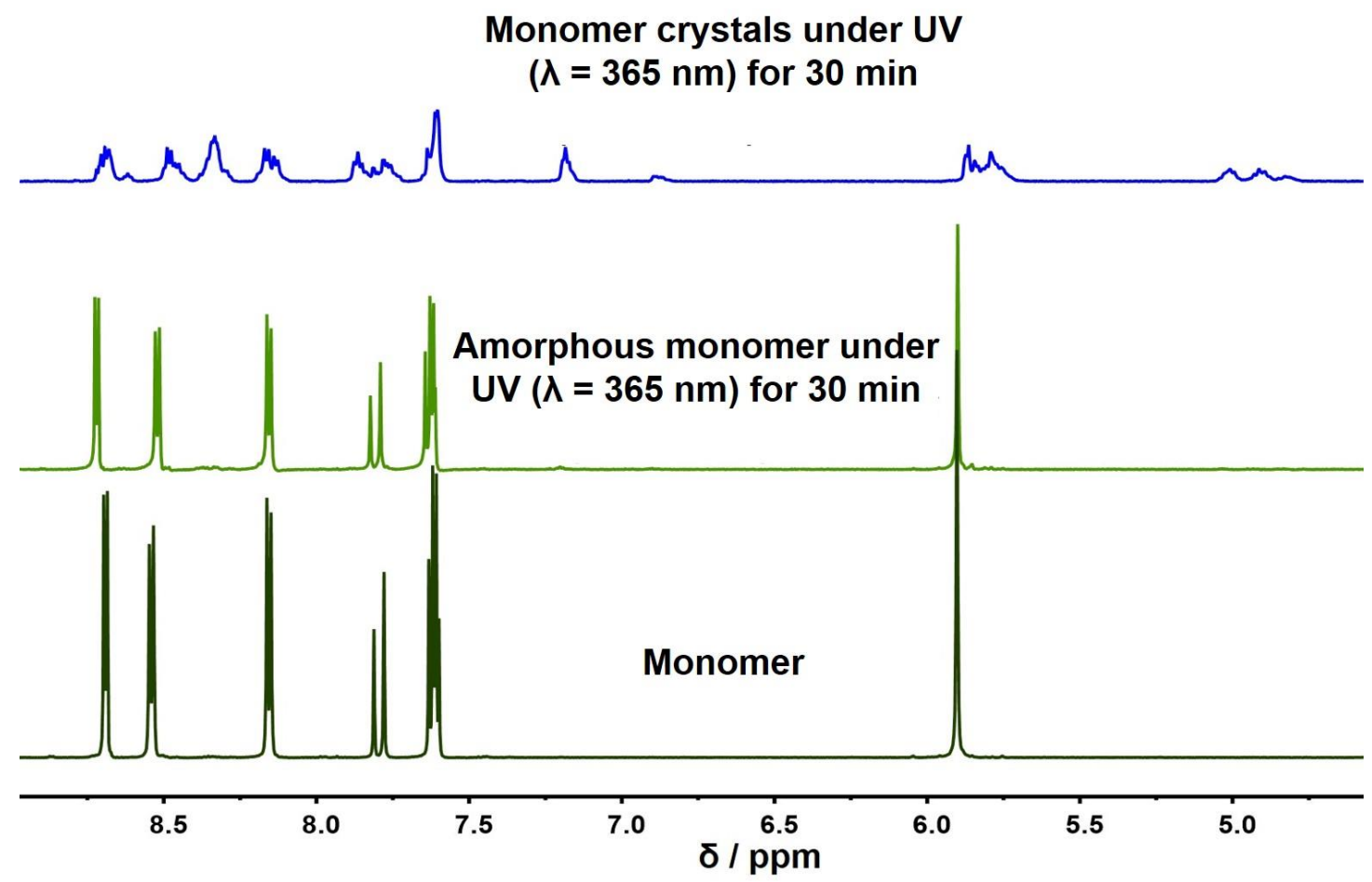

Figure S13. ${ }^{1} \mathrm{H}$ NMR spectroscopy of monomer (bottom), amorphous monomer under

UV for $3 \mathrm{~h}$ (middle) and monomer crystals under UV for $3 \mathrm{~h}$ (top). No notable changes in the ${ }^{1} \mathrm{H}$ NMR spectroscopy of amorphous monomer under UV for $3 \mathrm{~h}$. After irradiation for $3 \mathrm{~h}$ under the same conditions, the monomer crystals, however, polymerized. This result indicates that the pre-assembly of monomer in crystals is essential for the topochemical photopolymerization to occur. 


\section{In-Situ Powder X-Ray Diffractometer}
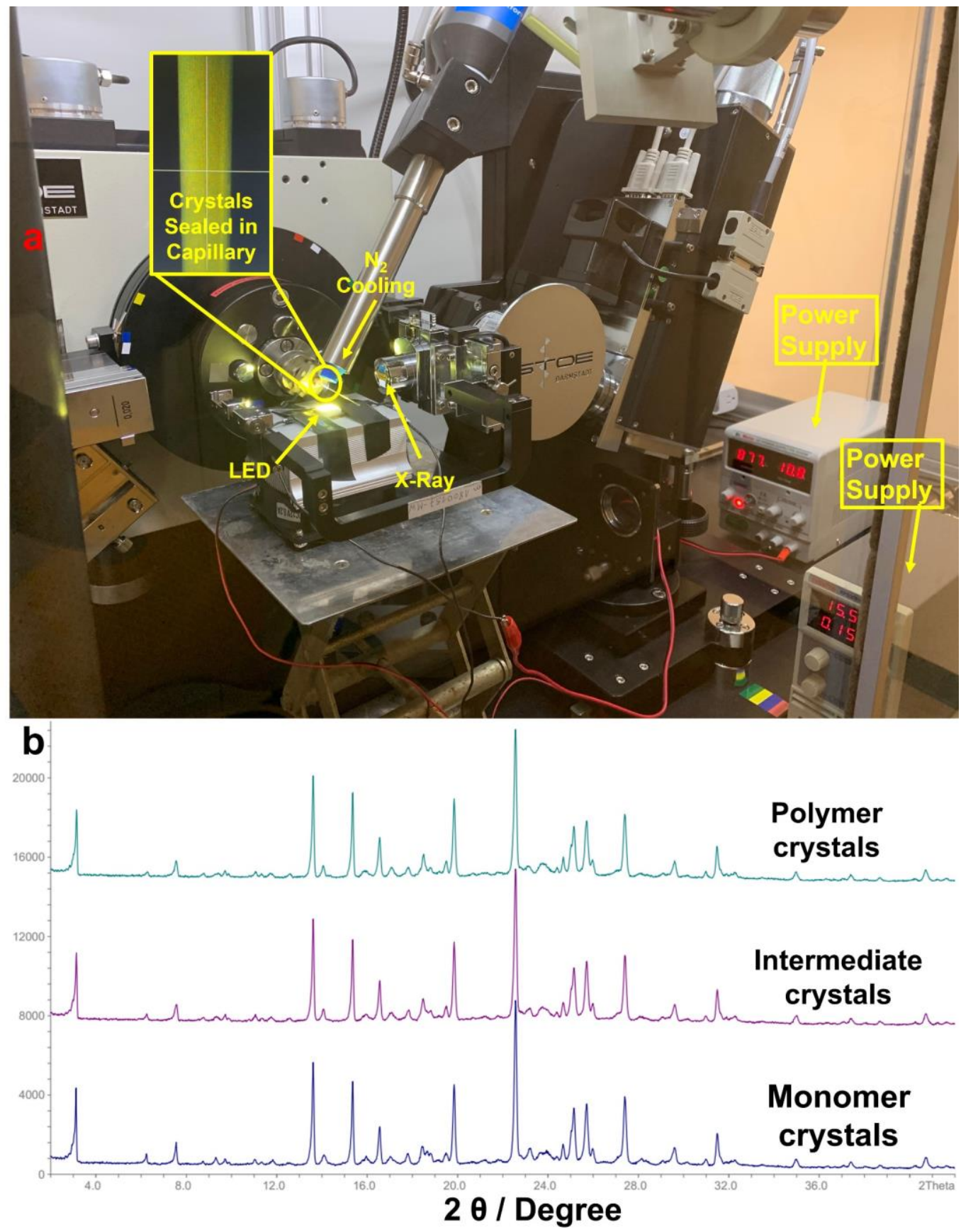

Figure S14. In-situ powder X-ray diffractometer (PXRD) for monitoring the photopolymerization. a, The setup of in-situ PXRD. The monomer crystals were sealed in a capillary and irradiated by UV continuously $(0-13 \mathrm{~h})$ at below $100 \mathrm{~K}$. b, PXRD patterns for crystal samples of monomer, intermediate and polymer at $100 \mathrm{~K}$. There were no obvious differences between the PXRD patterns because the unit-cell parameters are slightly different. 


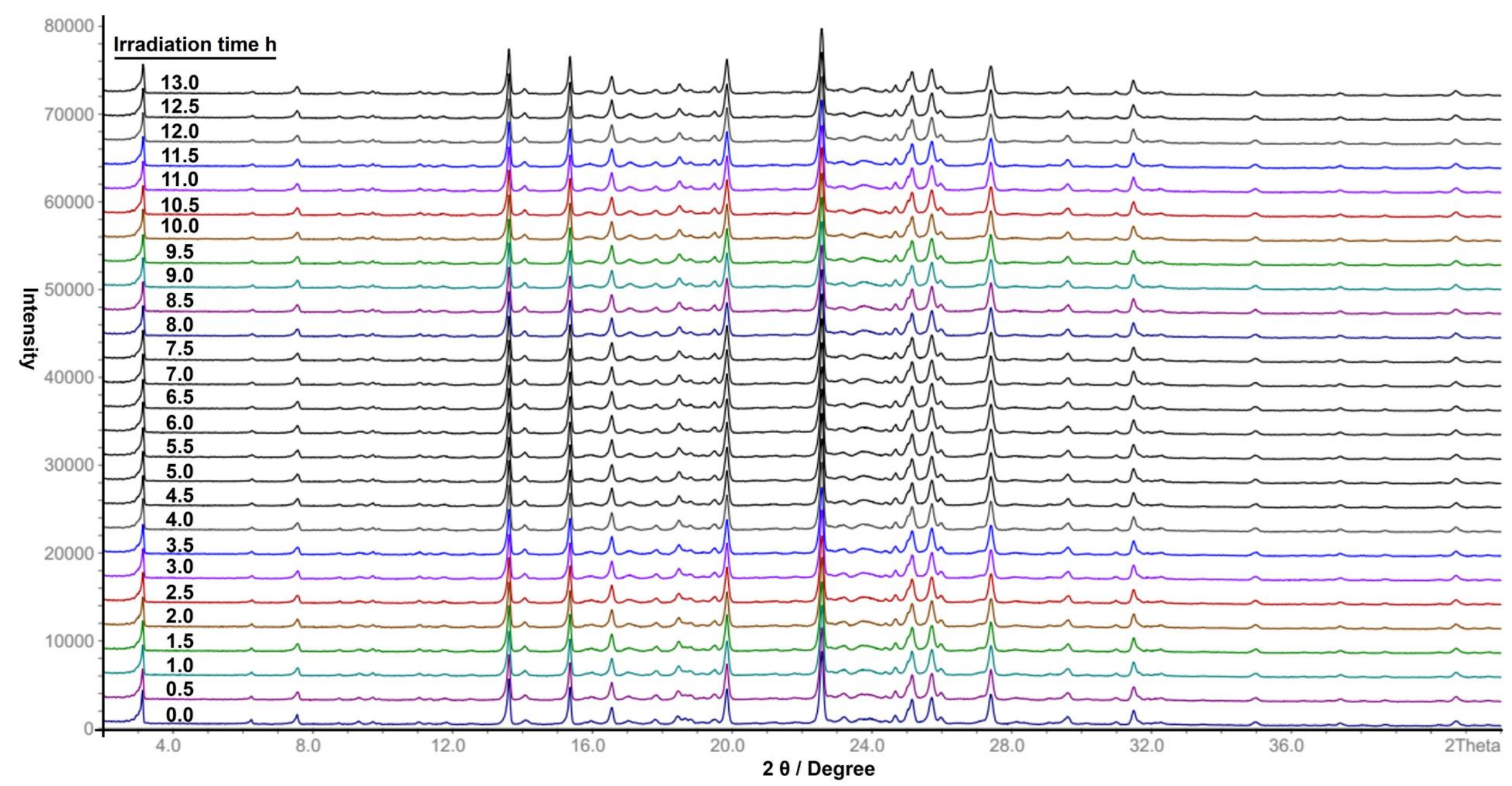

Figure S15. In-situ PXRD patterns for monitoring the photopolymerization at $100 \mathrm{~K}$. There were no virtual changes during the polymerization because the unit-cell parameters changed slightly. 


\section{Section E. Scanning Electron Microscopy (SEM)}
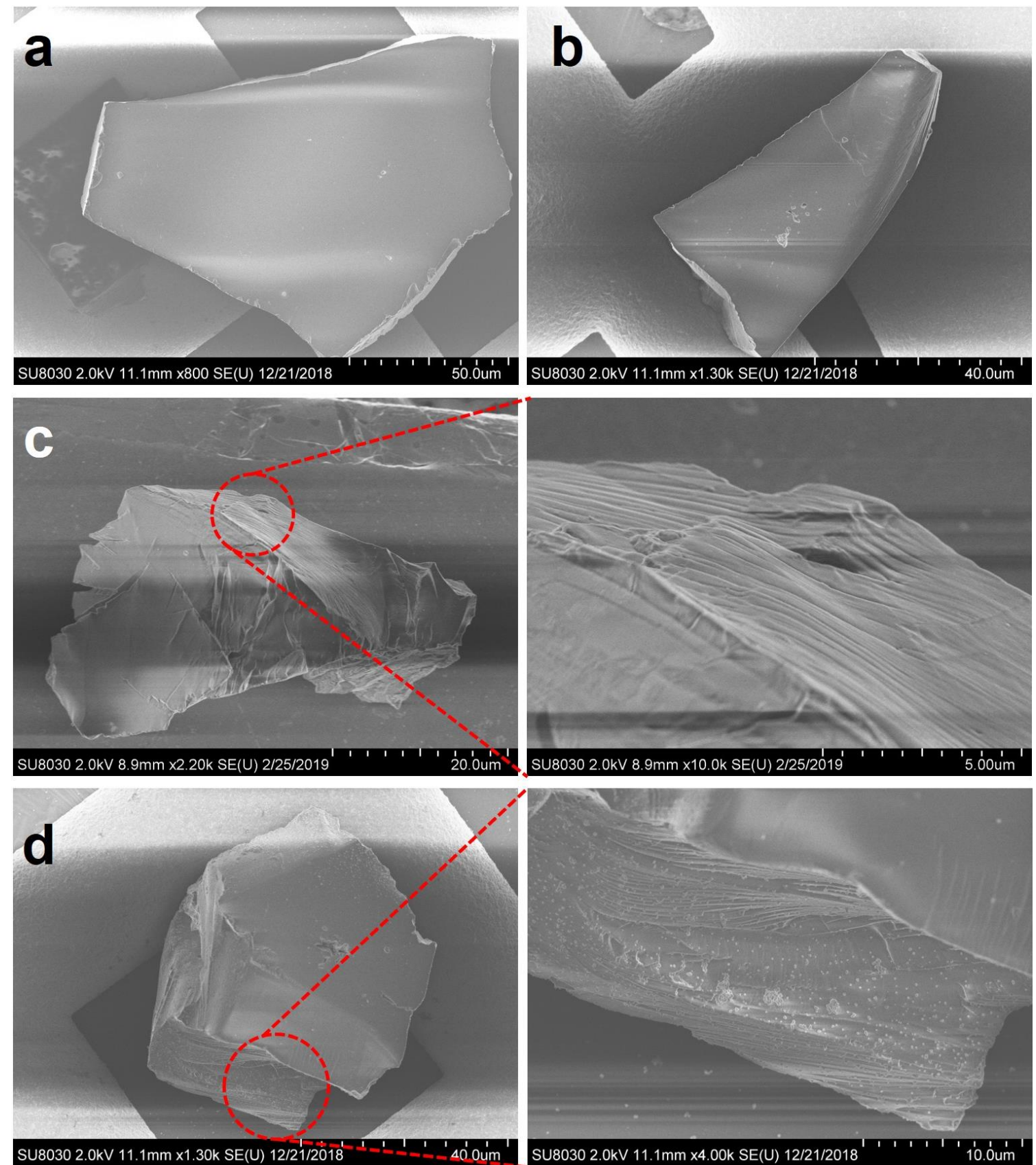

Figure S16. SEM images. a and b, The apparently smooth surfaces of plate-like polymeric crystals. $\mathbf{c}$ and $\mathbf{d}$, The laminar structures were clearly shown in the red circles. 


\section{Section F. Transmission Electron Microscopy (TEM)}

In order to prepare samples with appropriate sizes for (HR)TEM imaging, the polymeric crystals were partially exfoliated. The crystals were stirred at room temperature for $1 \mathrm{~h}$ and sonicated for $30 \mathrm{~min}$ in $\mathrm{MeCN}$. Then, they were deposited on a $\mathrm{Cu}$ grid.
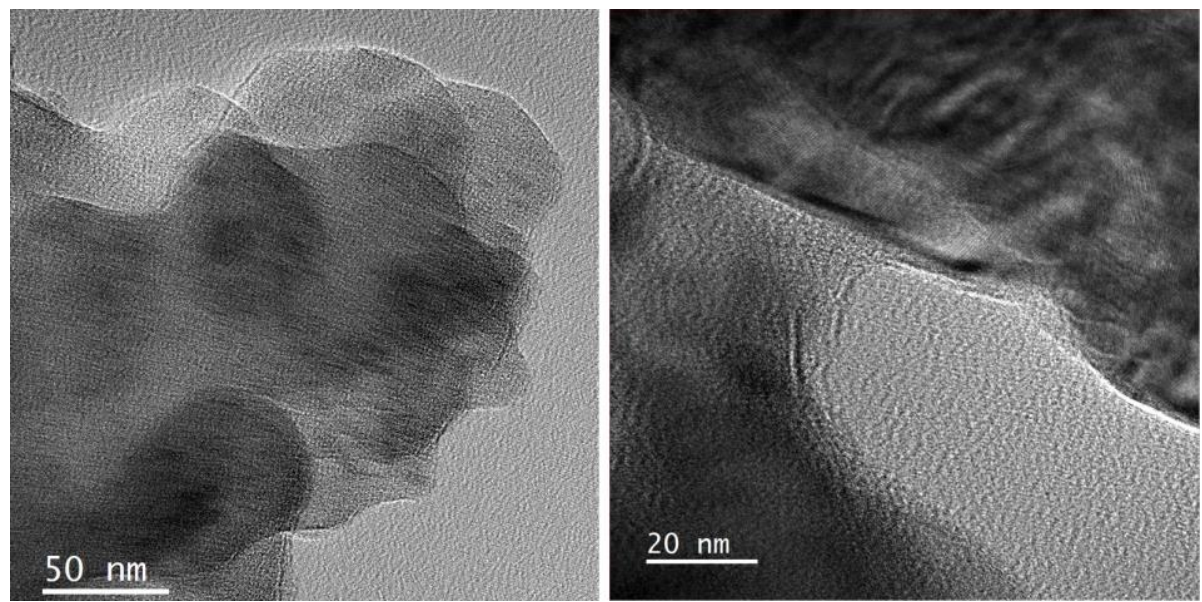

Figure S17. Low magnification TEM images. The laminar structures were clearly shown at the edges of the sample

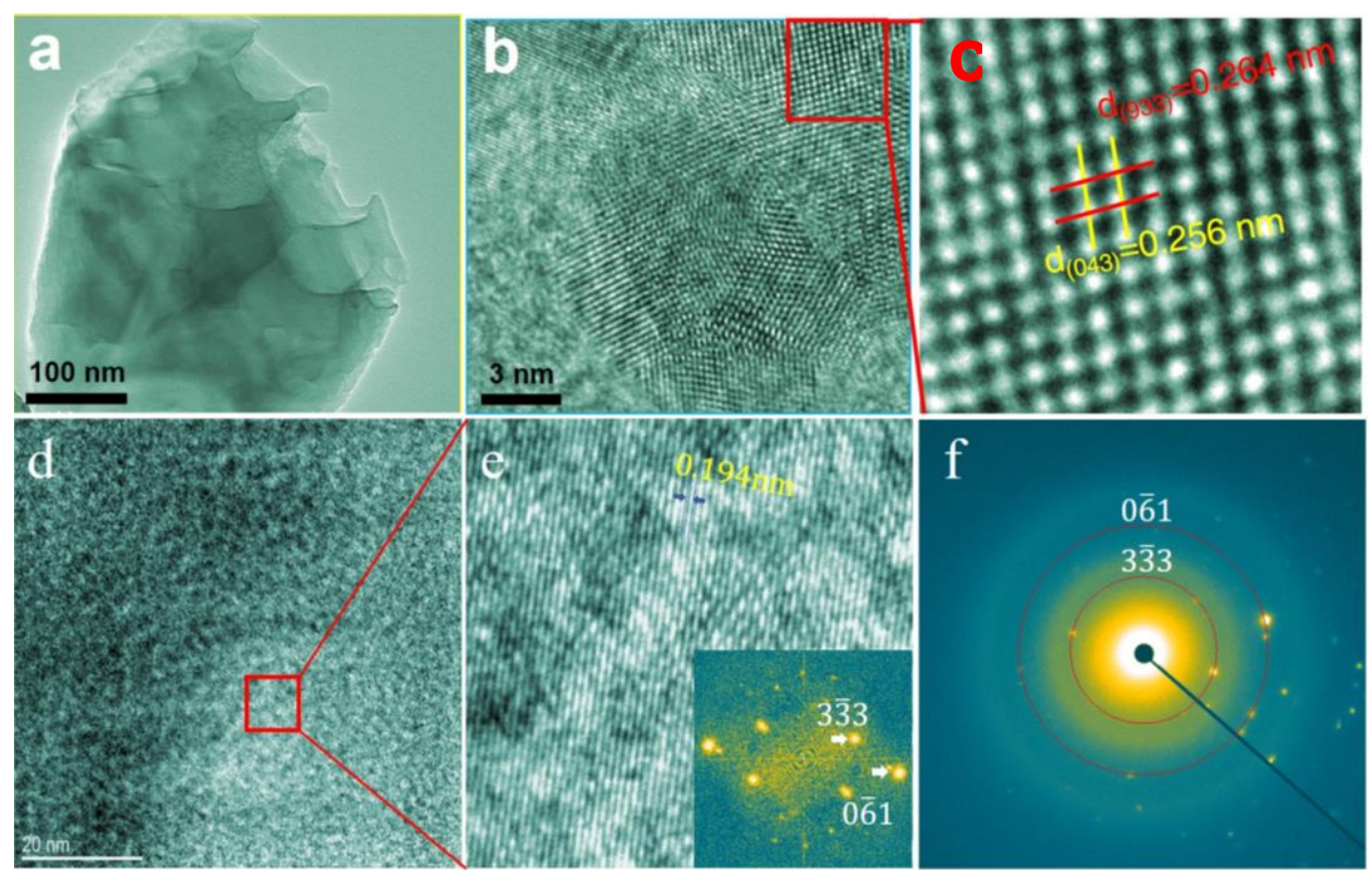

Figure S18. HRTEM images with distinct crystal lattice fringes and welldistinguishable selected area electron diffraction (SAED). a, HRTEM images of bulk samples with distinct crystal lattice fringes (b and $\mathbf{c})$. d, HRTEM images of exfoliated samples with distinct crystal lattice fringes (e) and SAED (f). 


\section{Section G. Exfoliation}

Liquid-phase exfoliation has been explored in order to achieve a thorough exfoliation. The ionic polymer crystals were stirred in $\gamma$-butyrolactone (GBL) under $323 \mathrm{~K}$ for 5 days. A micrometer-size monolayer with a height of monolayer $1.0 \mathrm{~nm}$ was afforded and characterized by AFM.

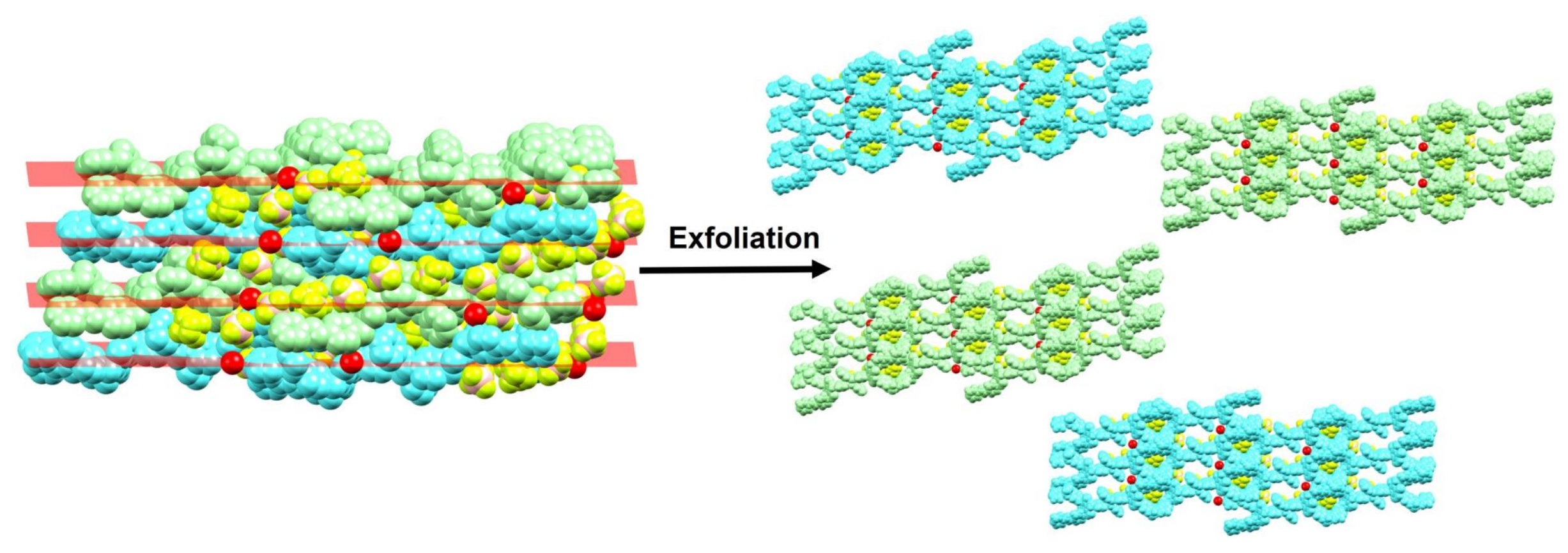

Figure S19. The schematic diagram for the exfoliation of polymer crystals to monolayer sheets 


\section{Section H. Nanoindentation}

Hardness and Young's moduli were measured on a Hysitron 950 Tribolndenter with a Berkovich indenter (radius $100 \mathrm{~nm}$ ). A large single crystal of monomer $\mathbf{1} \cdot 3 \mathrm{BF}_{4}$ was first mounted onto stainless steel atomic force microscopy specimen disks by epoxy (J-B Kwik) with the (001) plane facing up. For all measurements, the loading and unloading rate were kept at about $50 \mu \mathrm{N} / \mathrm{s}$ and before unloading, the indenter was held at constant load $(500 \mu \mathrm{N})$ for $10 \mathrm{~s}$. The same crystal was checked before and after polymerization. The data were analyzed using standard Oliver and Pharr analysis to extract the reduced moduli and hardness. The out-of-plane Young's moduli of the materials can be further derived with a Young's modulus $E$ of $1141 \mathrm{GPa}$ and Poisson's ratio $v$ of 0.07 for the diamond tip and $v=0.3$ for monomer and polymer crystals. For each type of sample, two crystals were prepared and 17 indentations were performed on each crystal. The reported values for each type of sample were averaged by all 34 measurements.
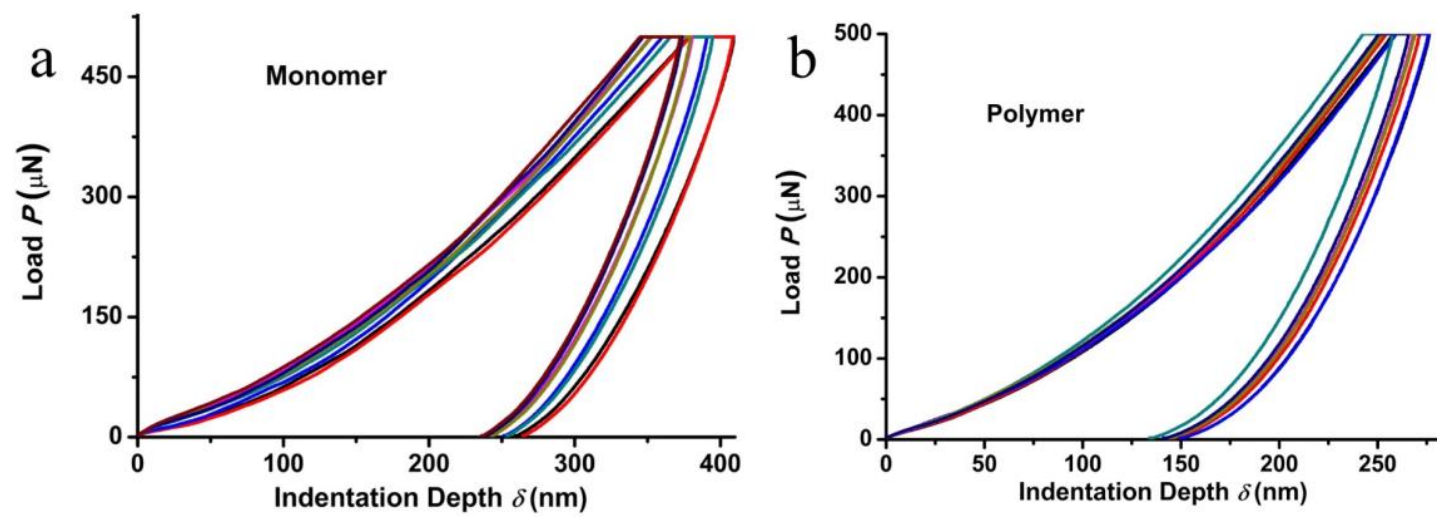

Figure S20. Eight typical nanoindentation curves performed on the monomer (a) and polymer (b) crystals. 

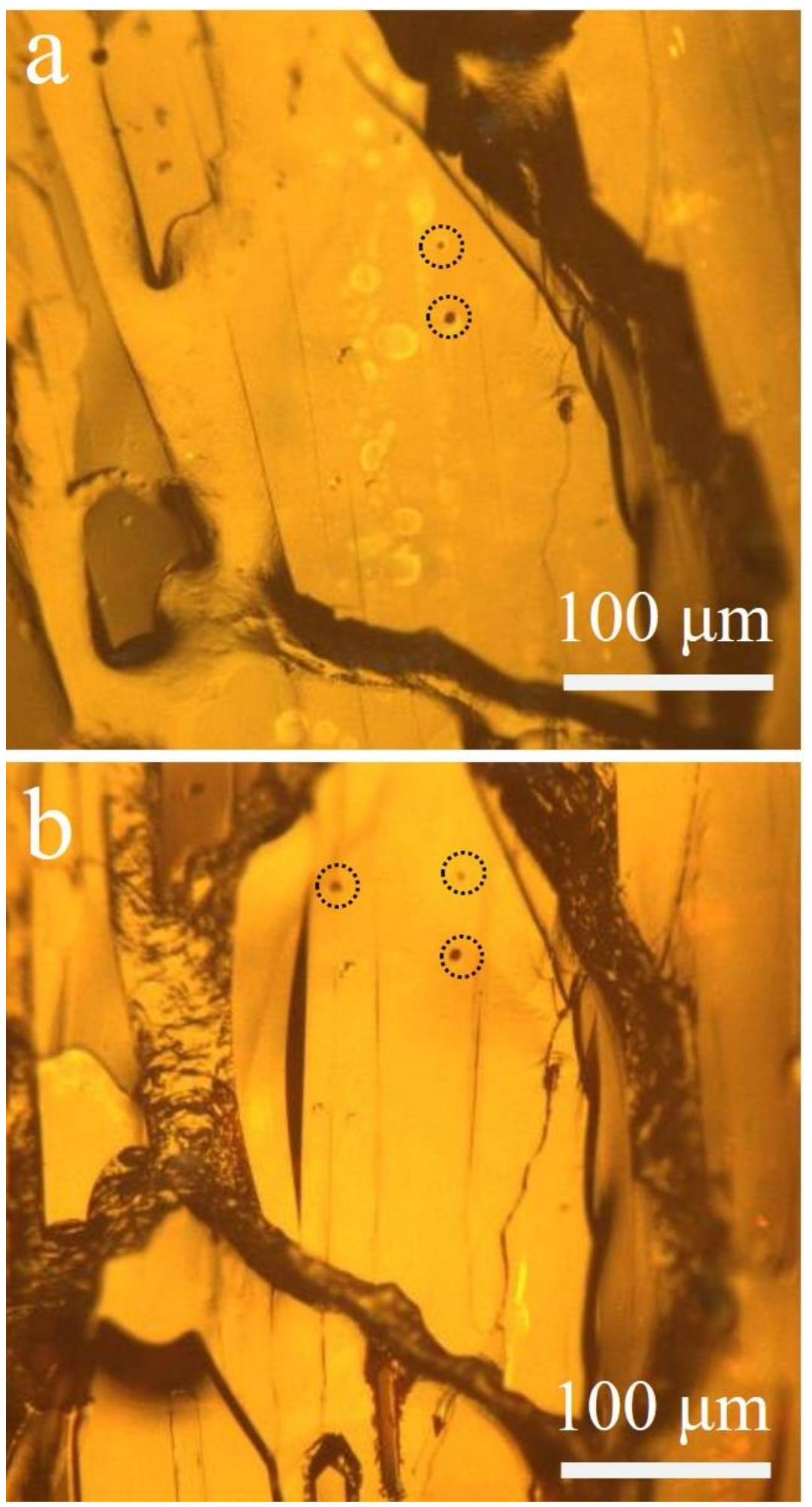

Figure S21. Pictures of crystals for nanoindentation. a, Monomer crystals and b, polymer crystals (from the irradiation of the same monomer crystals for $12 \mathrm{~h}$ ). The holes labeled by black dashed circles are imprints from the indenter after nanoindentation measurement. 


\section{Section I. DFT Calculations and Response Properties}

The anisotropic single-crystal properties of the ionic polymer crystal were computed using first-principles density functional theory (DFT) calculations performed using the periodic CRYSTAL17 code $^{8}$. The B3LYP hybrid exchange-correlation functional ${ }^{9-11}$ was used with a semiempirical dispersion correction accounting for two-body and three-body contributions (B3LYP-D3) ${ }^{12}$, and the geometry optimization was compared with another commonly used DFT functional, PBE-D3 ${ }^{12-14}$. Each DFT calculation was performed with all-electron atom-centered Gaussian-type basis sets of double-zeta quality, similar to previous work on the dielectric and electronic properties of other supramolecular and framework materials ${ }^{15,16}$. The crystalline orbitals were considered as linear combinations of Bloch functions (BF) and evaluated using a regular threedimensional (3D) mesh in reciprocal space. Each BF was constructed from local atomic orbitals (AOs), consisting of linear combinations of Gaussian-type functions (GTF). The all-electron basis sets contained a total of 9,528 basis functions, corresponding to 3,984 electrons spread over 3,384 shells per unit cell for the polymer crystal.

Due to the structural disorder of the anions present in the experimentally obtained CIF, the symmetry was reduced in the geometry optimization to allow for the lowest energy ordered structure to be obtained. The geometry optimization resulted in symmetry reduction from the $C 2 / c$ (15) to $P 2 / c$ (13) space group. The lattice parameters and atomic coordinates of the ionic polymer crystal were optimized, while maintaining the $P 2 / c$ (13) space group symmetry. The optimization was considered to have converged when the maximum and root-mean-square (RMS) gradient, and the maximum and RMS 
atomic displacements were simultaneously below $4.5 \times 10^{-4}, 3.0 \times 10^{-4}, 1.8 \times 10^{-3}$ and $1.2 \times 10^{-3}$ a.u., respectively.

The static dielectric constant and refractive index of the ionic polymer crystal were calculated analytically via a Coupled-Perturbed Hartree-Fock/Kohn-Sham (CPHF/CPKS) approach with the B3LYP-D3 functional. The CPHF/CPKS approach involved calculating the polarizability and dielectric tensors, as reported in Ref.15. The electronic band gap of the ionic polymer crystal is predicted to be $3.72 \mathrm{eV}$ using the B3LYP-D3 functional, and the values of the other anisotropic properties are summarized in Table S2.

For the calculation of the anisotropic Young's moduli of the ionic polymer crystal, reported in Table S3 and Scheme S2, the methodology reported in Ref. S17 was used with a minimal basis Hartree-Fock (HF) composite method (HF-3C) instead of DFT ${ }^{18}$. The method was designed to compete with semi-empirical tight-binding approaches without neglecting many-center integrals and has been tested using the CRYSTAL17 $\operatorname{code}^{18}$. It is worth noting that the HF-3C results reported here are limited to Young's moduli and a full analysis of the anisotropic mechanical properties and stability will be reported in a follow-on study.

Summarized in Table S2 are the comparisons of the optimized lattice parameters calculated from DFT and HF-3C (for ideal crystalline structures) with the experimental values. 
Table S2. Comparison of the lattice parameters for the polymer crystal.

\begin{tabular}{|c|c|c|c|c|}
\hline \hline \multicolumn{5}{|c|}{ Polycationic Polymer Crystal } \\
\hline \multirow{2}{*}{ Method } & $a$ & $b$ & $c$ & $\beta$ \\
\cline { 2 - 5 } & $57.282(7)$ & $11.7334(15)$ & $16.2714(18)$ & $104.674(7)$ \\
\hline $\begin{array}{c}\text { Experimental } \\
\text { CIF }\end{array}$ & 57.98 & 11.05 & 16.40 & 105.0 \\
\hline B3LYP-D3 & 58.23 & 11.13 & 16.50 & 104.9 \\
\hline PBE-D3 & 57.82 & 10.95 & 16.51 & 102.9 \\
\hline \multicolumn{5}{|c|}{ HF3C } \\
\hline $\begin{array}{l}\text { The average difference compared to the experimental parameters for B3LYP-D3: } \\
\text { 2.05\% }\end{array}$ \\
$\begin{array}{l}\text { The average difference compared to the experimental parameters for PBE-D3: } 2.11 \% \\
\text { The average difference compared to the experimental parameters for HF3C: } 2.69 \%\end{array}$ \\
\hline
\end{tabular}

Table S3. Summary of the anisotropic properties of the polycationic polymer crystal

\begin{tabular}{ccccc}
\hline $\begin{array}{c}\text { Crystallographic axis } \\
\kappa\end{array}$ & $\boldsymbol{a}$ & $\boldsymbol{b}$ & $\boldsymbol{c}$ \\
\hline $\begin{array}{c}\text { Dielectric tensor, } \\
n\end{array}$ & 1.92 & 2.04 & 2.45 \\
\hline & 1.39 & 1.43 & 1.57 \\
\hline
\end{tabular}

Scheme S2. Anisotropic 3D representation of the Young's moduli (in GPa) of the polymer crystal. 
Section J. Environmental and Chemical Stabilities

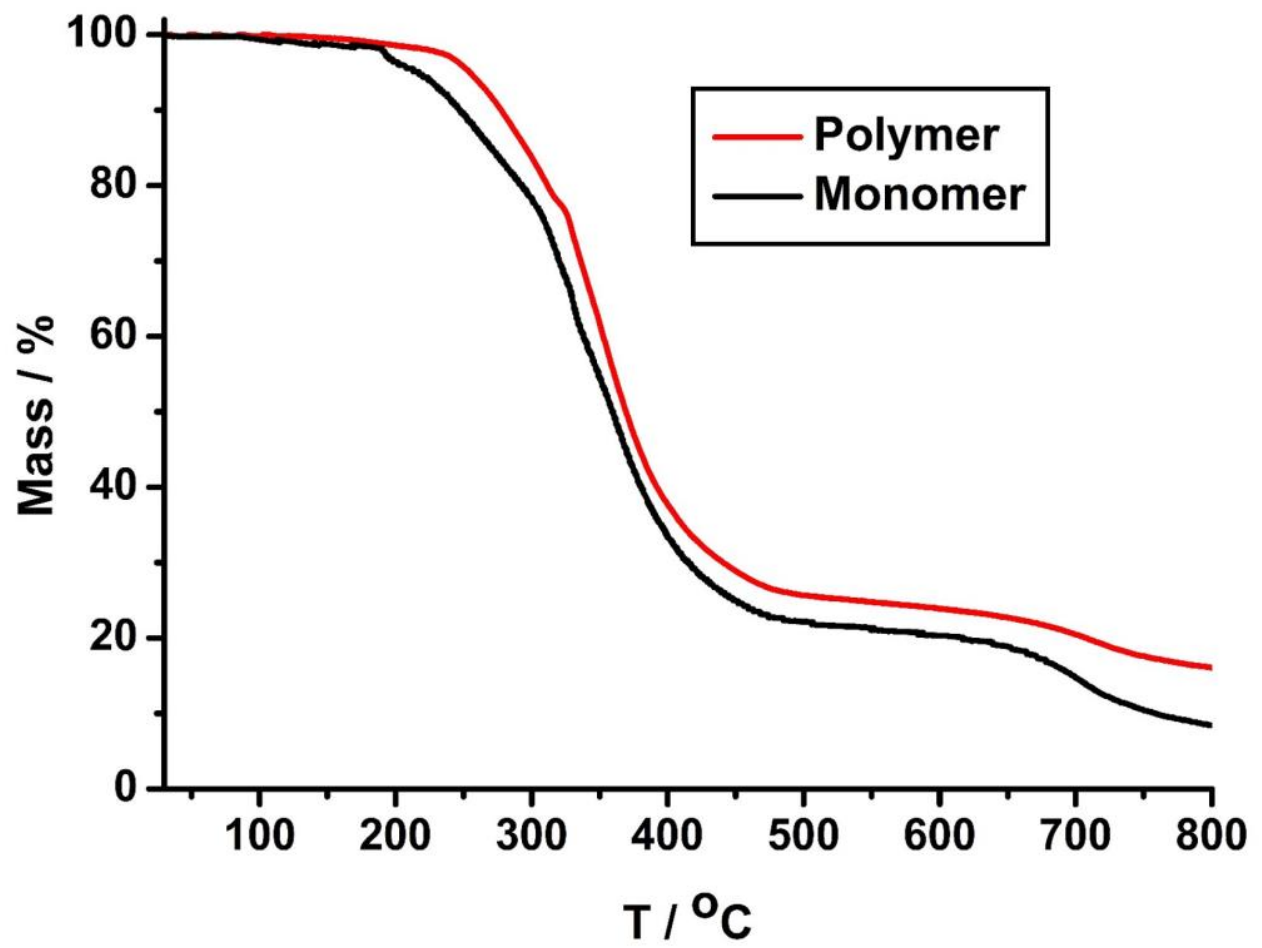

Figure S22. TGA Curves of monomer and polymer under $\mathrm{N}_{2}$. The decomposition temperatures of monomer and polymer are 463 and $513 \mathrm{~K}$.

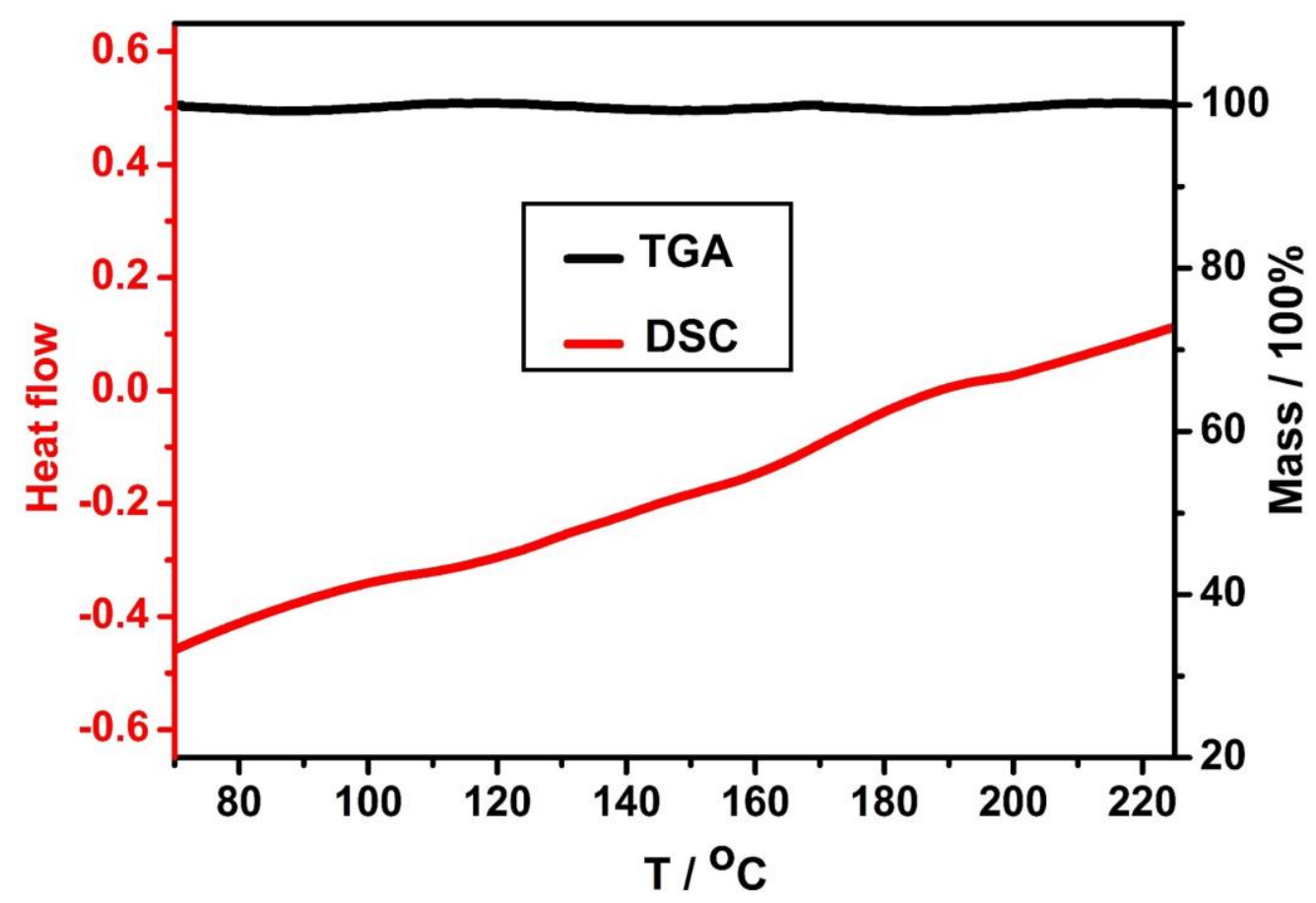

Figure S23. TGA and DSC curves of the polymer under $\mathrm{N}_{2}$. No any phase transitions or depolymerization before the decomposition of the polymer. 


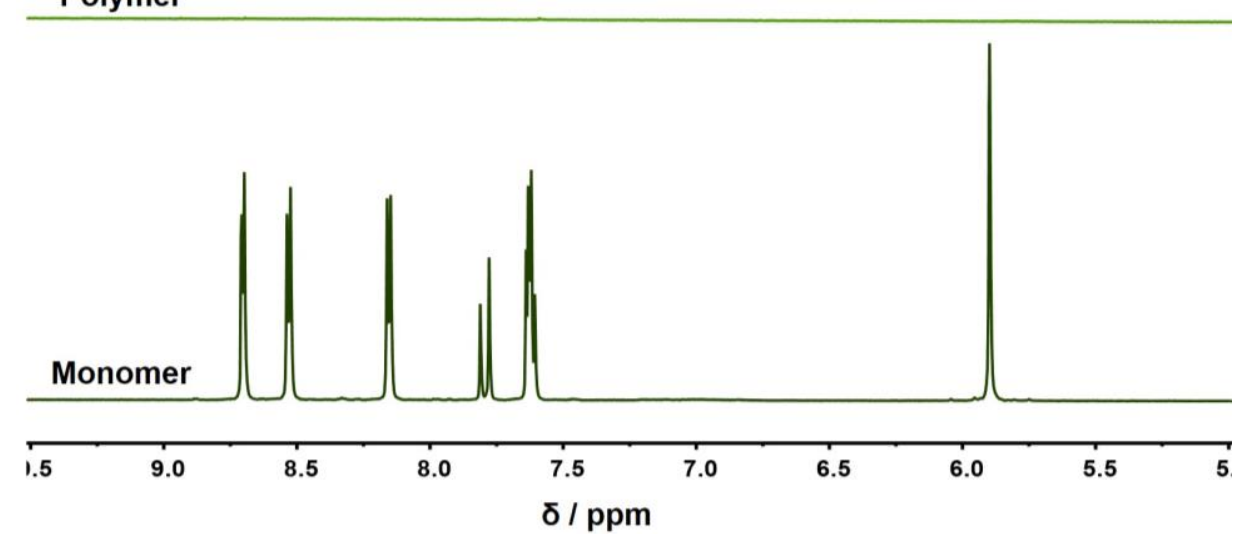

Figure S24. ${ }^{1} \mathrm{H}$ NMR Spectra of monomer, polymer and polymer after DSC measurement (bottom-to-top). There is no detectable degradation of the polymer samples, which were heated up to $503 \mathrm{~K}$ during the DSC, measurement in the ${ }^{1} \mathrm{H}$ NMR spectra.

Polymer irradiated under UV $(\lambda=254 \mathrm{~nm})$ for $36 \mathrm{~h}$

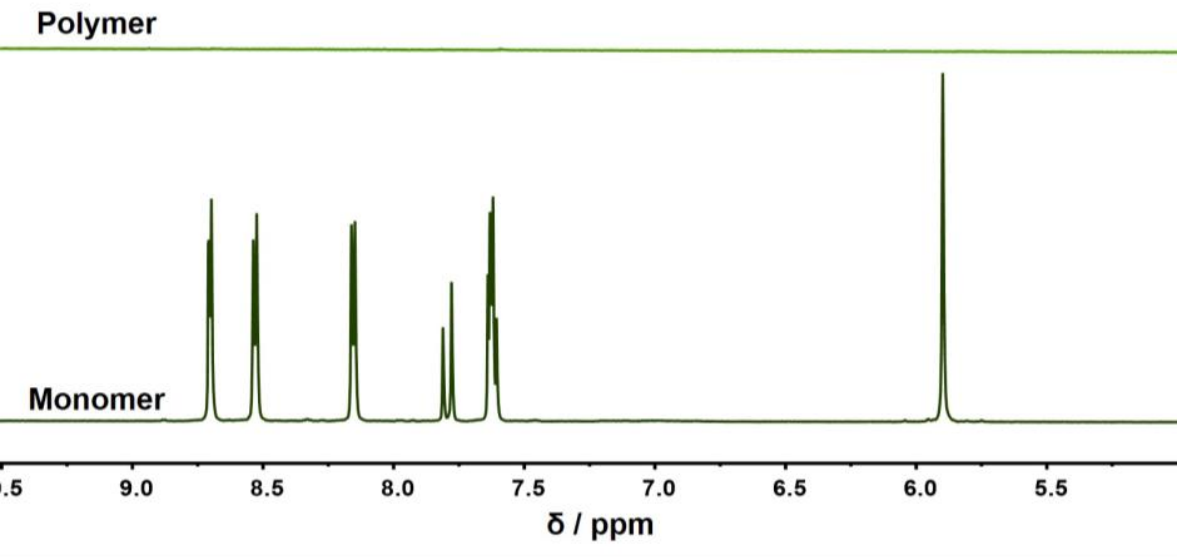

Figure S25. ${ }^{1} \mathrm{H}$ NMR spectra of monomer, polymer and polymer after irradiation under UV light $(\lambda=254 \mathrm{~nm})$ for $36 \mathrm{~h}$ (bottom to up). There no detectable degradation observed in the ${ }^{1} \mathrm{H}$ NMR spectra. 


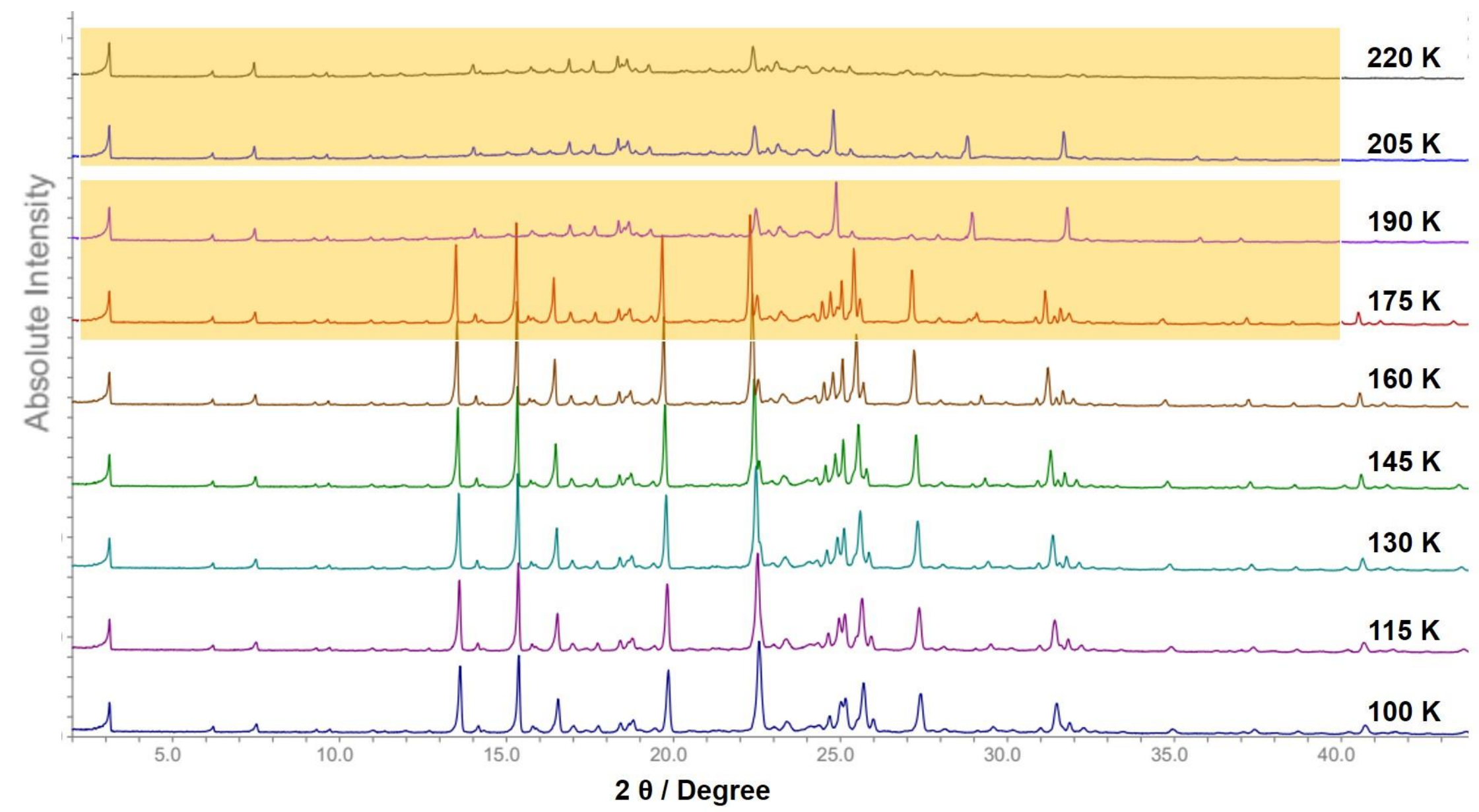

Figure S26. VT-PRD patterns of monomer $1 \cdot 3 \mathrm{BF}_{4}$ (from 100 to $220 \mathrm{~K}$ ). The changes of the patterns at 190 and $220 \mathrm{~K}$ can be ascribed to the melt of solvents during the measurement (the melting points of $i \mathrm{Pr}_{2} \mathrm{O}$ and $\mathrm{MeCN}$ are 187 and $225 \mathrm{~K}$, respectively). 


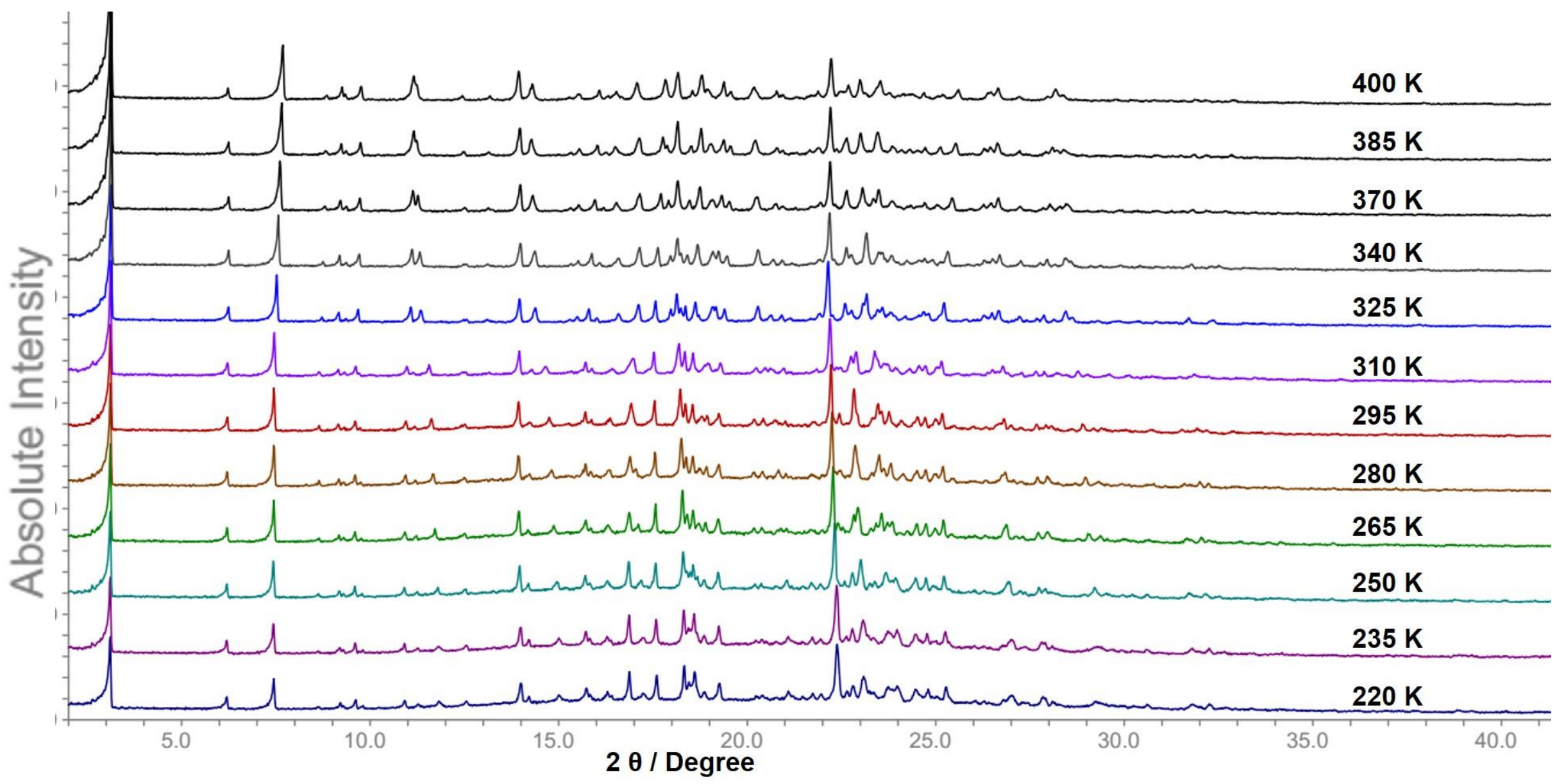

Figure S27. VT-PRD patterns of monomer $\mathbf{1}^{-3 \mathrm{BF}_{4}}$ (from 220 to $400 \mathrm{~K}$ ). Even when the crystals were heated up to $400 \mathrm{~K}$, the high crystallinity was still retained. 


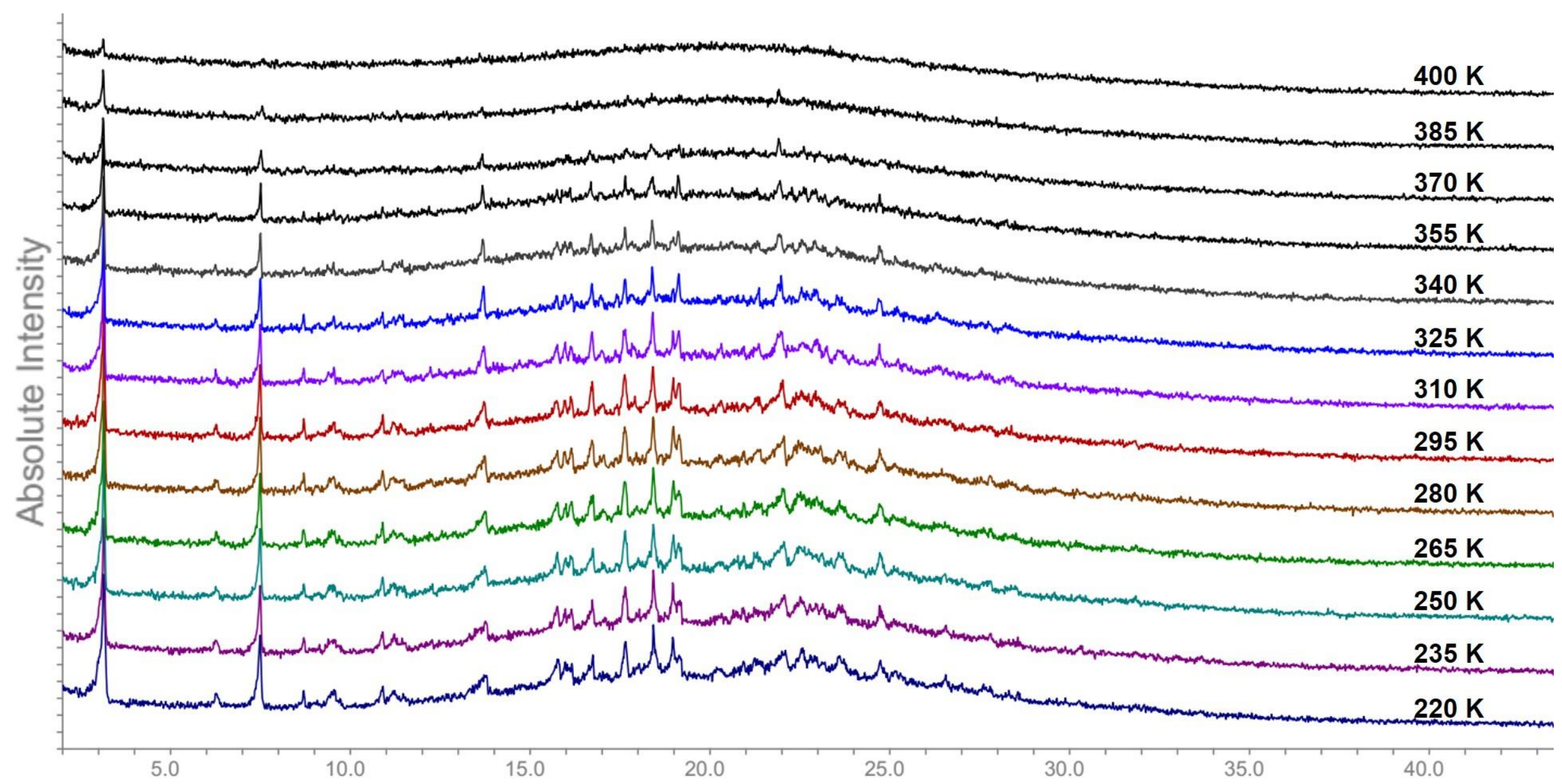

Figure S28. VT-PRD patterns of polymer $2 \bullet \mathrm{nBF}_{4}$ (from 220 to $400 \mathrm{~K}$ ). When heated to $400 \mathrm{~K}$, the polymer crystals lost their crystallinity. After the SCSC topochemical polymerization, the quality of the crystals decreased. 

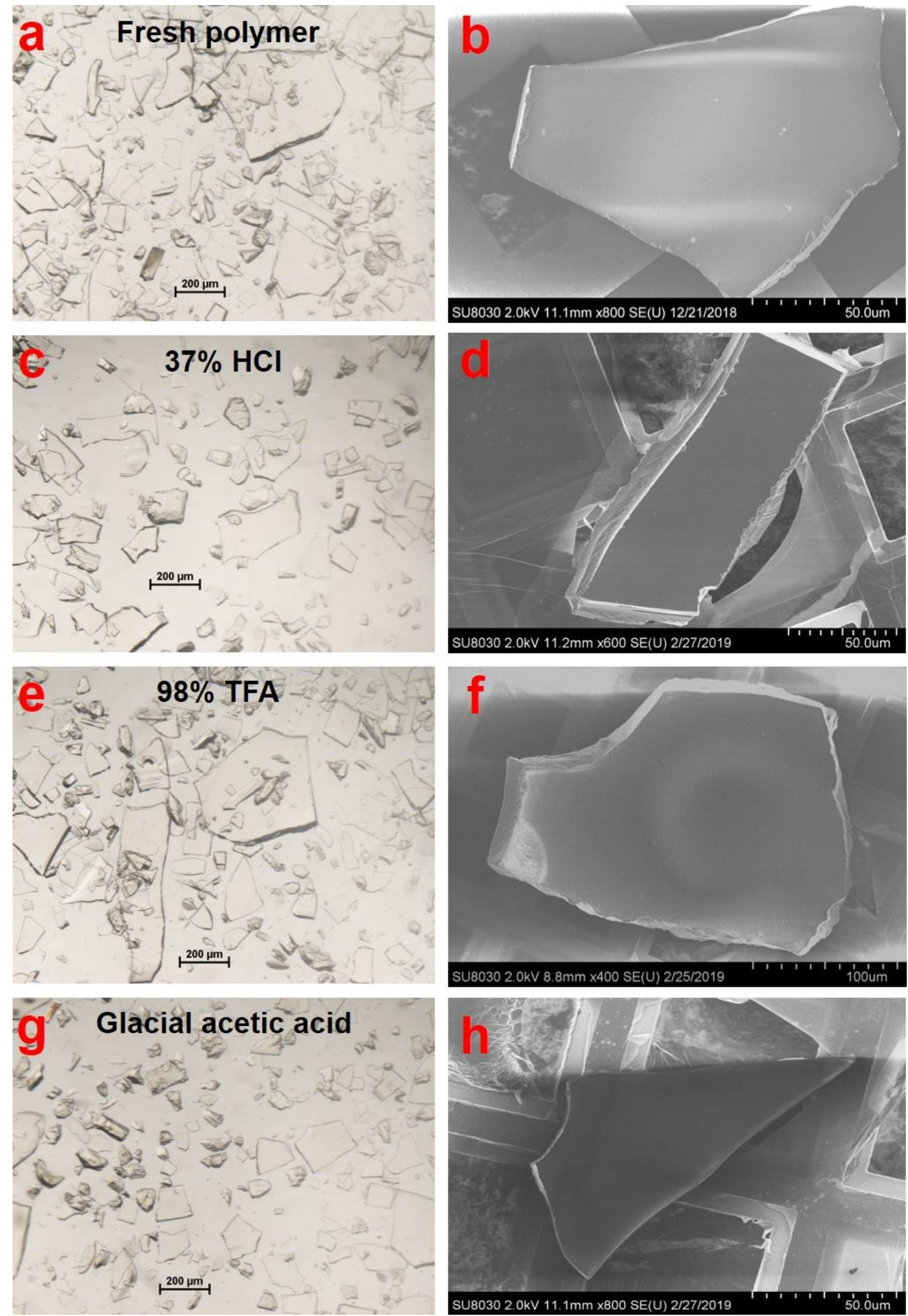

Figure S29. Optical microscope image and SEM images of the freshly prepared polymer samples (a and $\mathbf{b})$ and polymers soaked in various concentrated acids $(\mathbf{c}-\mathbf{h})$ for more than 3 months. Their morphology and physical integrity are retained. 


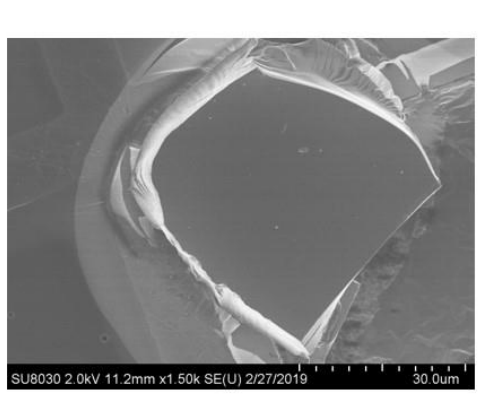

B Ka1_2

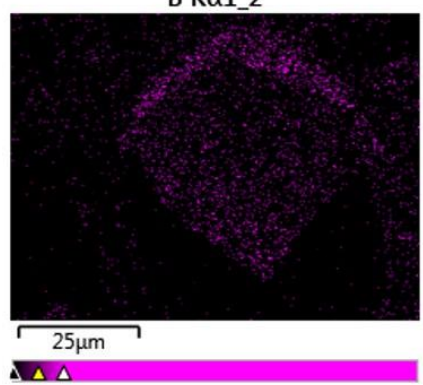

C K $\alpha 1 \_2$

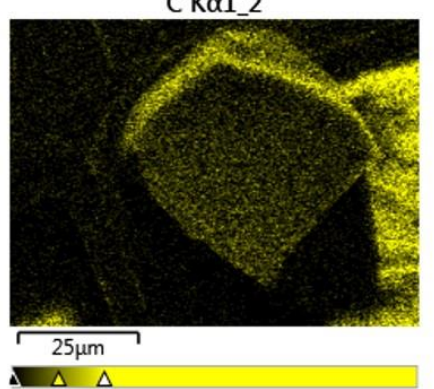

$\mathrm{O} K \alpha 1$

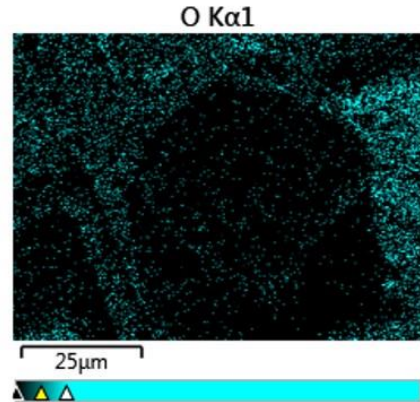

$\mathrm{N} \mathrm{K \alpha 1 \_ 2}$

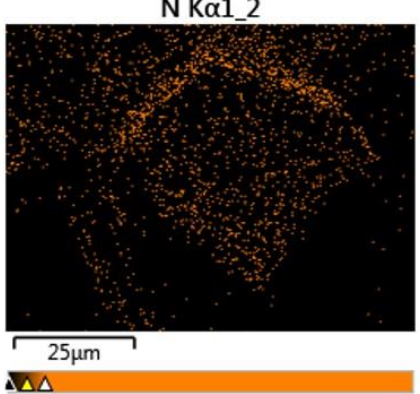

$\mathrm{Cl} \mathrm{K \alpha 1}$

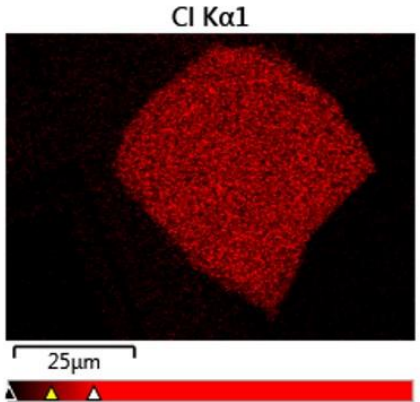

Figure S30. SEM images and EDS maps of a polymer sample soaked in $37 \%$ hydrochloric acid solution for 3 months. The sample retains the smooth facet, plate morphology and chemical compositions. SEM-EDS Maps show the homogeneity of the samples with all the original elements $(\mathrm{C} / \mathrm{N} / \mathrm{B})$ well-distributed within the samples. The presence of oxygen and chloride indicates that $\mathrm{H}_{2} \mathrm{O}$ and hydrochloric were adsorbed into the crystal.
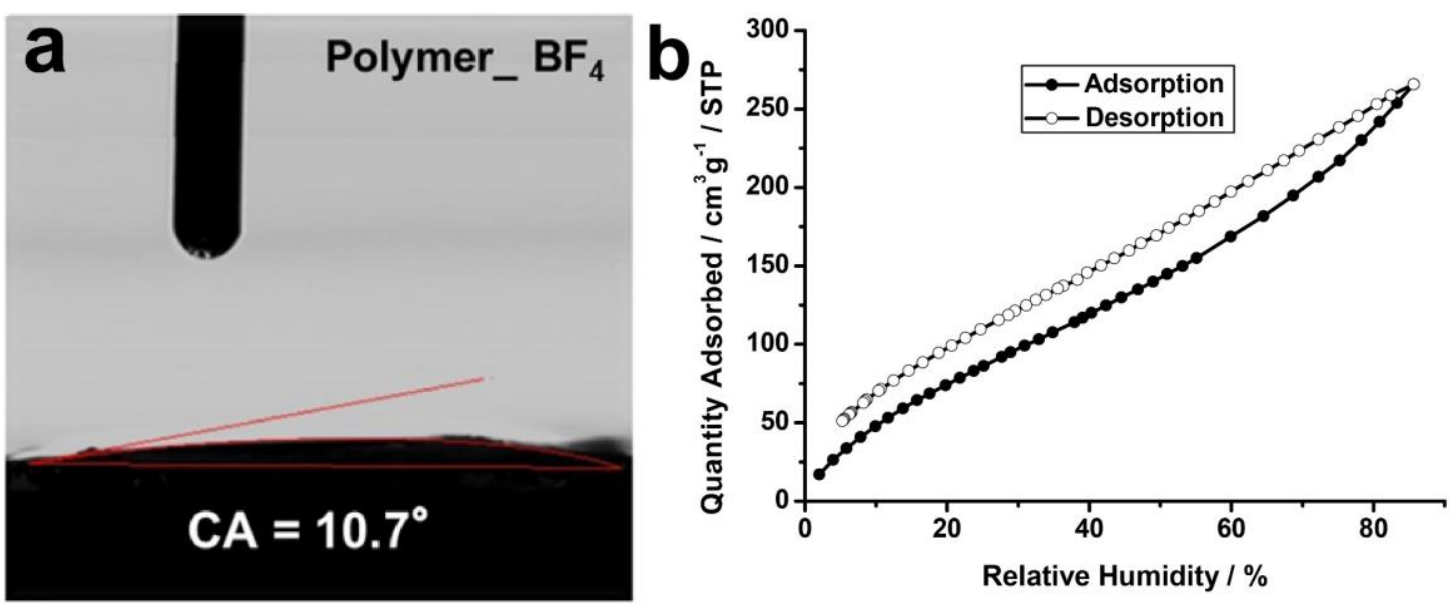

Figure S31. a, The $\mathrm{H}_{2} \mathrm{O}$ contact angle (CA) of a drop of $\mathrm{H}_{2} \mathrm{O}$ on the polymer. The polymer possesses a hydrophilic surface $\left(\mathrm{CA}=10.7^{\circ}\right)$. $\mathbf{b}$, The water absorption and desorption isotherms. 


\section{Section K. Proton Conductivity}

Samples were mechanically ground into sufficiently small particle sizes. Impedance data were recorded by Autolab PGSTAT128N between $100 \mathrm{kHz}$ and $0.1 \mathrm{~Hz}$ at $20 \mathrm{mV}$ amplitude, and analysized by Nova 2.0 software. A simple equivalent circuit was used here to simulate the Nyquist plots. Experiments were carried out in a home-made humidity control chamber with $\mathrm{N}_{2}$ atmosphere. Before each measurement, the sample was incubated for $2 \mathrm{~h}$ at different humidities to reach a stable status.

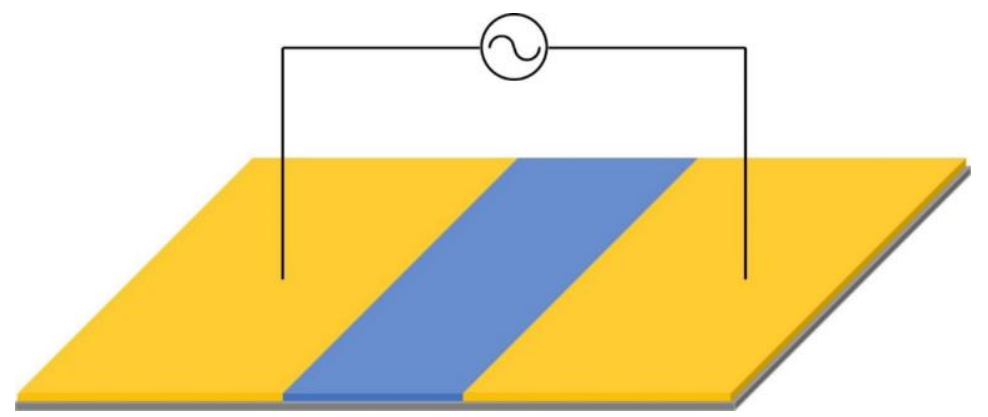

Figure S32. Two-terminal device used for EIS measurement. The two-terminal devices used in EIS measurements were fabricated on glass. Prior to device fabrication, the substrates were cleaned by sequential sonication in $\mathrm{Me}_{2} \mathrm{CO}$ and $i \mathrm{PrOH}$. Then, a $10 \mathrm{~nm}$ Titanium adhesion layer overlaid with a $100 \mathrm{~nm}$ gold was electron-beam evaporated onto the clean substrates through a shadow mask. The dimensions of the paired electrodes were $2.5 \mathrm{~cm}$ wide by $2.0 \mathrm{~cm}$ long with an inter-electrode separation of 50 $\mu \mathrm{m}$. The devices were completed by dropping cast the bulk polymer powders suspended in $\mathrm{MeCN}$ solution directly onto the electrode patterns, and the resulting films were allowed to dry in air overnight. 


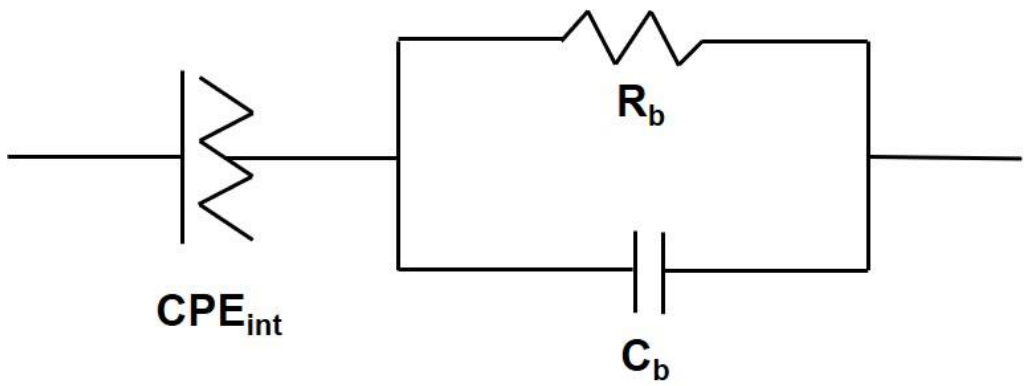

Figure S33. Equivalent circuit model used to fit the impedance data. A Constant Phase Element (CPE) was used to describe the non-ideal interface capacitance, $R_{\mathrm{b}}$ and $C_{\mathrm{b}}$ represent the resistance and capacitance of the sample, respectively.

The conductivity $(\sigma)$ of the sample is calculated with the equation below:

$$
\sigma=L / R_{\mathrm{b}} A
$$

Where $S$ and $L$ are the cross-sectional area and thickness of the sample, respectively, and $R_{\mathrm{b}}$ is the value of resistance, which was obtained from the impedance plots.

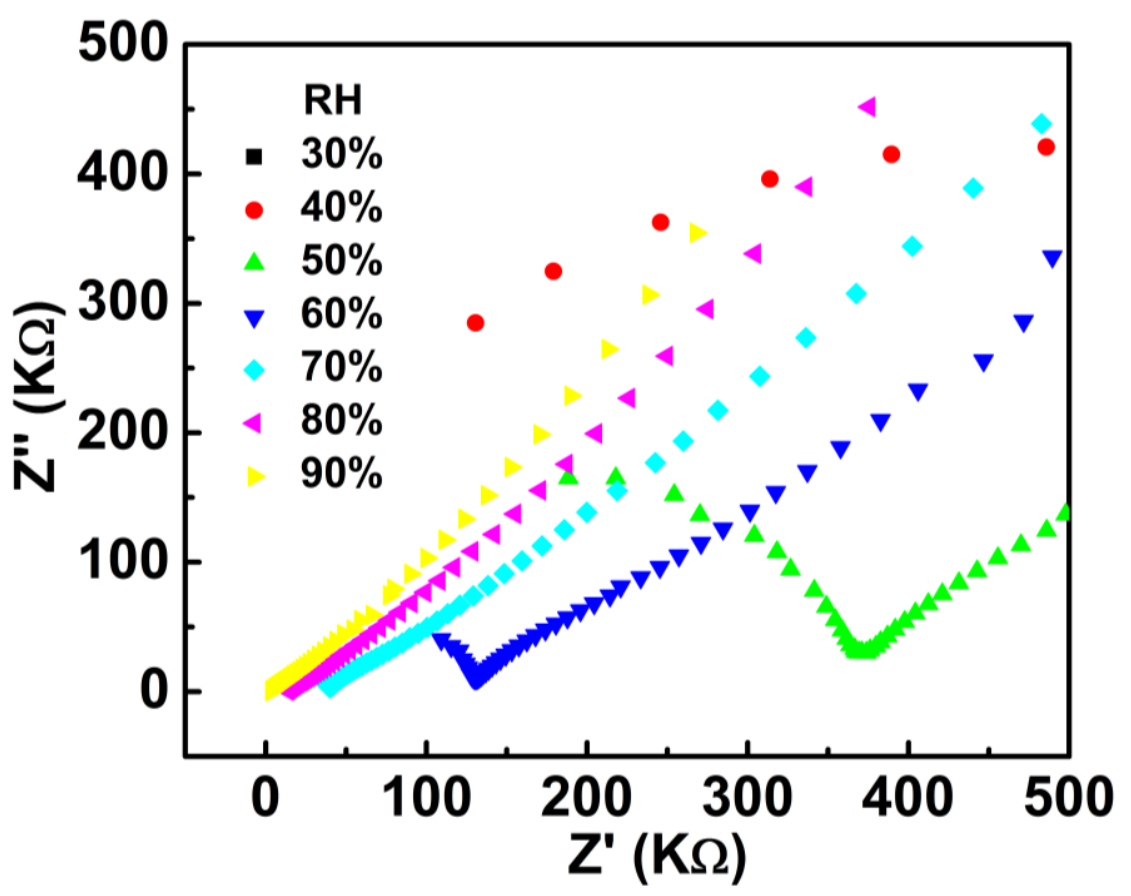

Figure S34. Nyquist plots showing the impedance of the polymer between $0.1 \mathrm{MHz}-$

$0.1 \mathrm{~Hz}$ at $298 \mathrm{~K}$ with varying RH. Zoom-in of Figure 5d. 


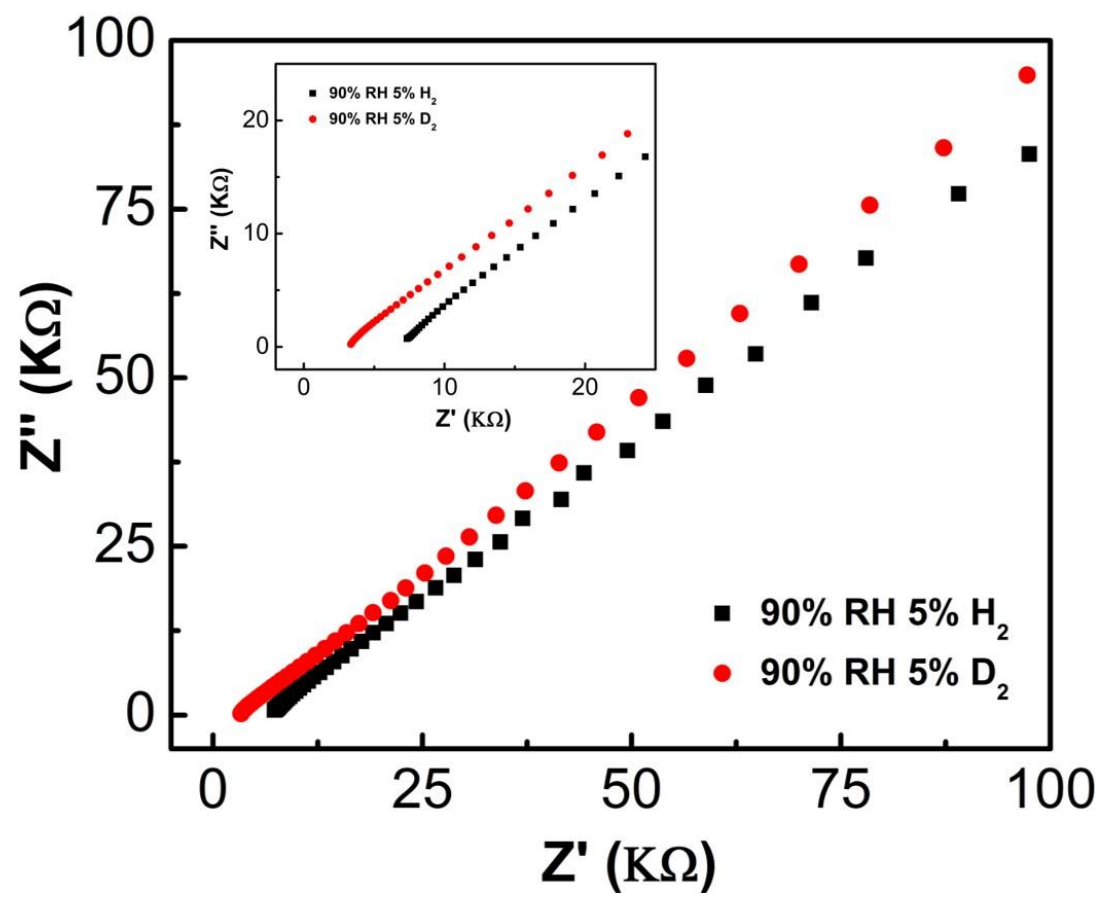

Figure S35. Kinetic isotope effect of the polymer at $90 \%$ RH. A Nyquist plot for the polymer-bridged two-terminal device in the presence of water vapour (black) and in the presence of deuterium oxide vapour (red). The conductivity calculated from the Nyquist plots are $3 \times 10^{-4} \mathrm{~S} \mathrm{~cm}^{-1}\left(\mathrm{H}^{+}\right)$and $1.4 \times 10^{-4} \mathrm{~S} \mathrm{~cm}^{-1}\left(\mathrm{D}^{+}\right)$, respectively.

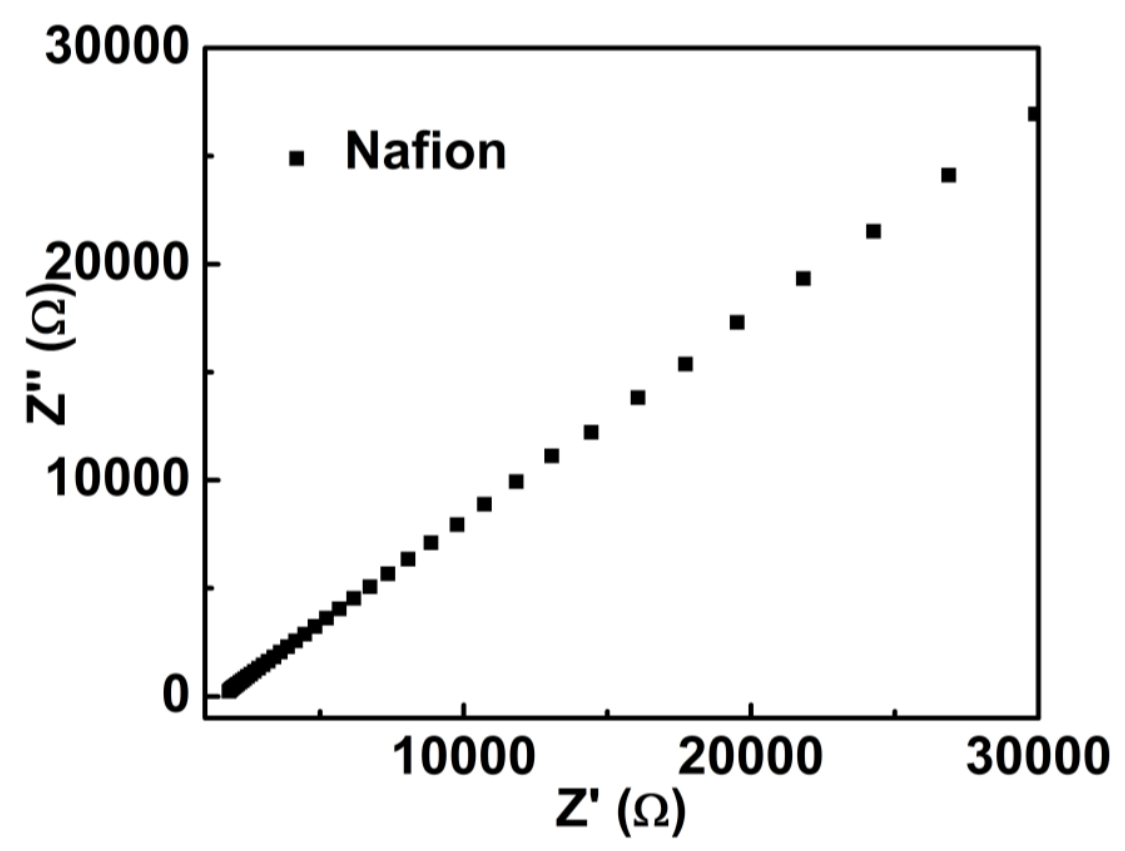

Figure S36. Nyquist plot of Nafion at $90 \% \mathrm{RH}$, yielding a value of $0.1 \mathrm{~S} \mathrm{~cm}^{-1}$. 
Molecular dynamics (MD) simulations of water structure were conducted using the RASPA software package ${ }^{19}$. The unit cell from the experimental crystal structure was expanded to a $1 \times 3 \times 2$ super-cell as the simulation box to satisfy the minimum image convention. In 6 different simulations, 10, 20, 30, 40, 50, or 60 water molecules per unit cell were added to the box by configuration-biased Monte Carlo insertions ${ }^{20}$ prior to the MD simulations. Then, 100 ps of MD in the canonical (NVT) ensemble at $298 \mathrm{~K}$ were conducted in each simulation in which the water molecules were allowed to move while the polymer and the counterions were held fixed. In all simulations, the LennardJones parameters for the polymer and counterions were taken from the DREIDING force field ${ }^{21}$, while the water molecules were described by the TIP4P model ${ }^{22}$. The partial charges of the polymer and counterion atoms were derived using the QEq method $^{23}$. The long-range van der Waals interactions were truncated at $12.8 \AA$ with analytical tail corrections, while the long-range electrostatics were calculated using the Ewald summation.

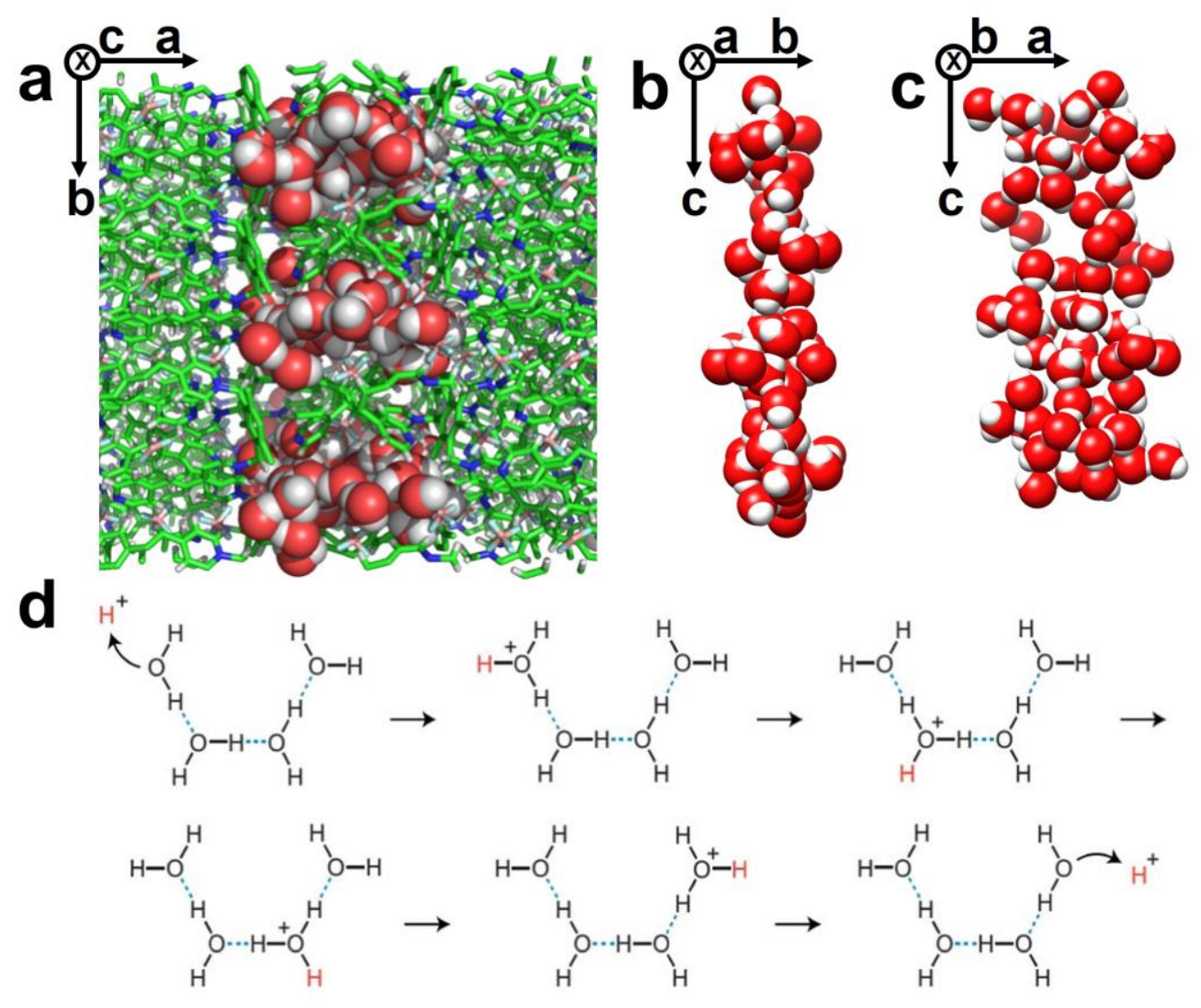

Figure S37. Simulated structure with 60 water molecules per unit cell and Grotthuss mechanism. a, Representation of simulated structure in which the 1D channels (going in to the page) are full of water molecules forming hydrogen-bonding networks. $\mathbf{b}$ and c, Water chains viewed from diffeent directions. d, the proposed Grotthuss mechanism for conduction of protons. 
Table S4. Comparison of proton conductivities of $2 \cdot n \mathrm{BF}_{4}$ with those of COFs.

\begin{tabular}{|c|c|c|c|}
\hline Material & $\begin{array}{l}\text { Proton conductivity } \\
\qquad\left(\mathrm{S} \mathrm{cm}^{-1}\right)\end{array}$ & $\begin{array}{c}\text { Relative Humidity } \\
(\mathrm{RH}) / \text { Temperature }\left({ }^{\circ} \mathrm{C}\right)\end{array}$ & Reference \\
\hline $2 \bullet \mathrm{nBF}_{4}$ & $2.6 \times 10^{-4}$ & $90 \% / 25$ & This work \\
\hline Nafion & $1 \times 10^{-1}$ & $90 \% / 25$ & This work \\
\hline NUS-10 (R) & $3.96 \times 10^{-2}$ & $97 \% / 25$ & ACS Appl. Mater. Interfaces. 2016, 8, 18505. \\
\hline LiCl@RT-COF-1 & $6.45 \times 10^{-3}$ & $100 \% / 40$ & J. Am. Chem. Soc. 2017, 139, 10079. \\
\hline EB-COF:PW 12 & $3.32 \times 10^{-3}$ & $97 \% / 25$ & J. Am. Chem. Soc. 2016, 138, 5897. \\
\hline PA@TP-Azo & $9.9 \times 10^{-4}$ & $98 \% / 60$ & J. Am. Chem. Soc. 2014, 136, 6570. \\
\hline RT-COF-1AcB & $5.25 \times 10^{-4}$ & $100 \% / 40$ & Nat. Commun. 2015, 6, 6486. \\
\hline RT-COF-1AC & $1.07 \times 10^{-4}$ & $100 \% / 40$ & J. Am. Chem. Soc. 2017, 139, 10079. \\
\hline PA@TP-Stb & $2.3 \times 10^{-5}$ & $98 \% / 60$ & J. Am. Chem. Soc. 2014, 136, 6570. \\
\hline RT-COF-1 & $1.83 \times 10^{-5}$ & $100 \% / 40$ & J. Am. Chem. Soc. 2017, 139, 10079. \\
\hline aza-COF-2 & $8.78 \times 10^{-6}$ & $97 \% / 50$ & Chem. Mater. 2019, 31, 819. \\
\hline EB-COF:Br & $2.82 \times 10^{-6}$ & $97 \% / 25$ & J. Am. Chem. Soc. 2016, 138, 5897. \\
\hline PA@TpBpy-МC & $2.5 \times 10^{-3}$ & $0 \% / 100$ & J. Mater. Chem. A 2016, 4, 2682. \\
\hline im@TPB-DMTPCOF & $1.79 \times 10^{-3}$ & $0 \% / 125$ & Nat. Mater. 2016, 15, 722. \\
\hline Phytic@TpPa-(SO3H-Py) & $5 \times 10^{-4}$ & $0 \% / 120$ & Chem. Mater. 2016, 28, 1489. \\
\hline $\mathrm{TpPa}_{-} \mathrm{SO}_{3} \mathrm{H}$ & $1.7 \times 10^{-5}$ & $0 \% / 120$ & Chem. Mater. 2016, 28, 1489. \\
\hline
\end{tabular}




\section{Section L. NMR Spectroscopy}
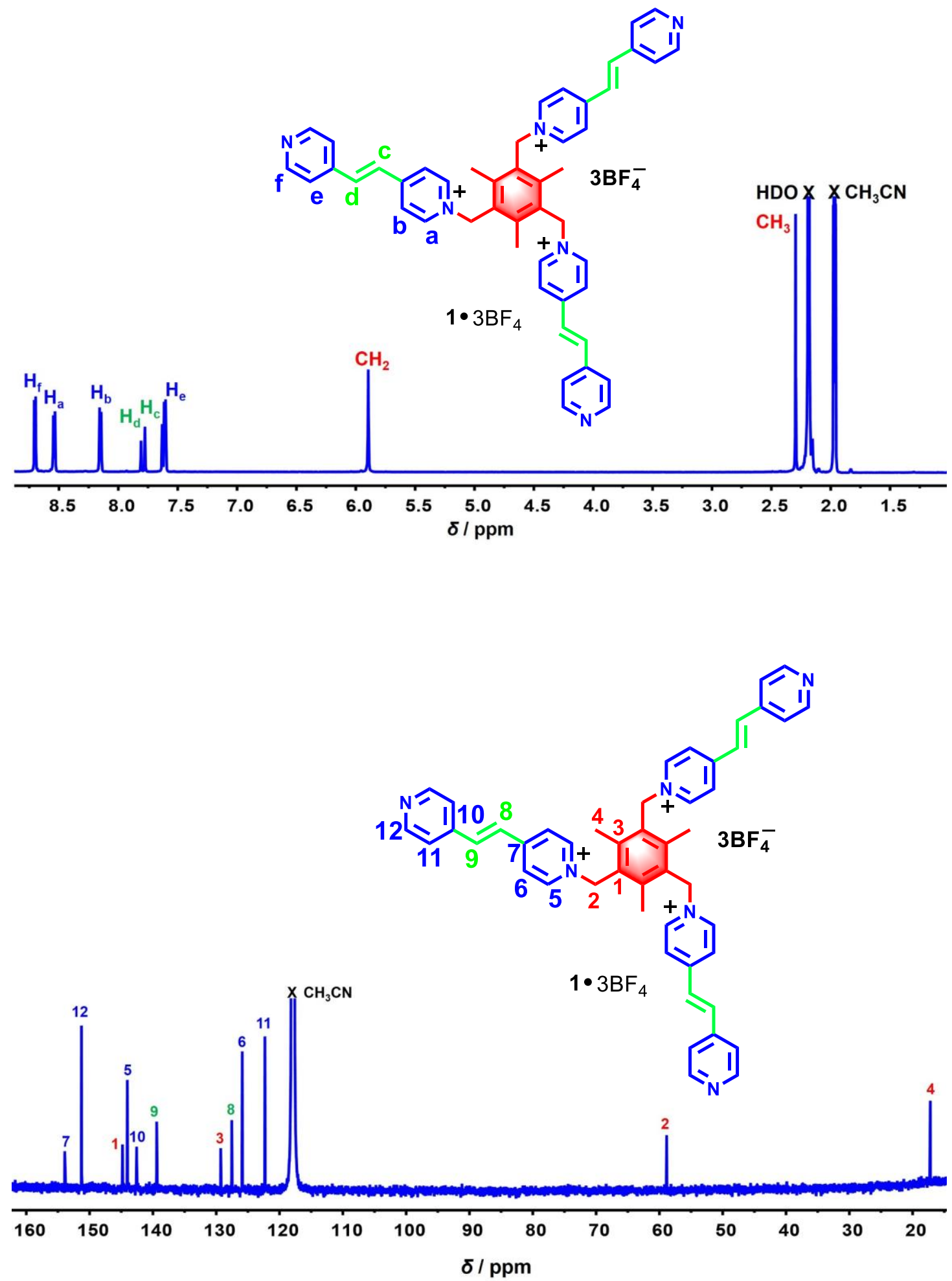

Figure S38. ${ }^{1} \mathrm{H}$ (top) and ${ }^{13} \mathrm{C}$ (bottom) NMR Spectra of $\mathbf{1} \cdot 3 \mathrm{BF}_{4}$. 

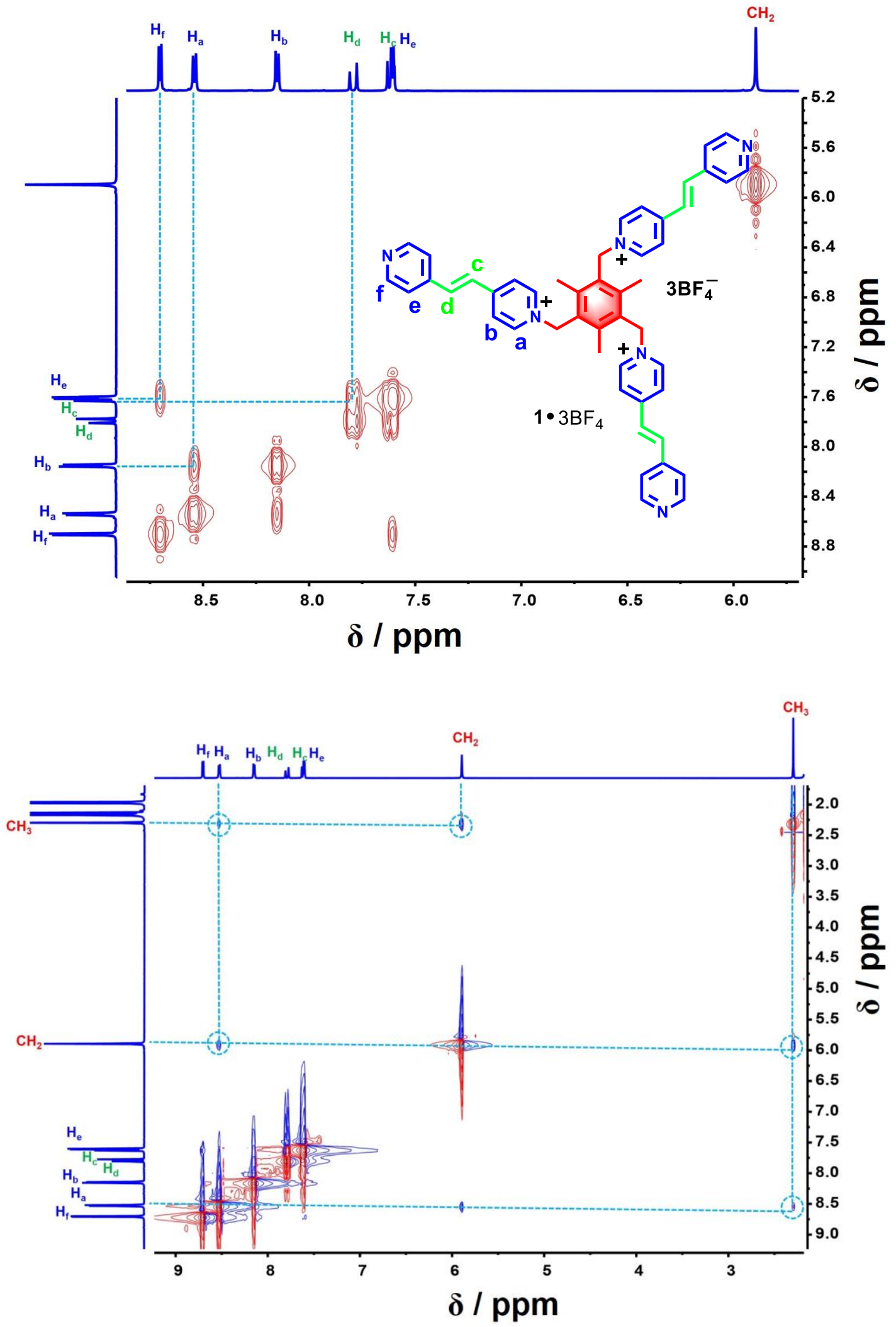

Figure S39. H-H COSY (top) and H-H NOSY (bottom) NMR spectra of $\mathbf{1} \cdot 3 \mathrm{BF}_{4}$. 

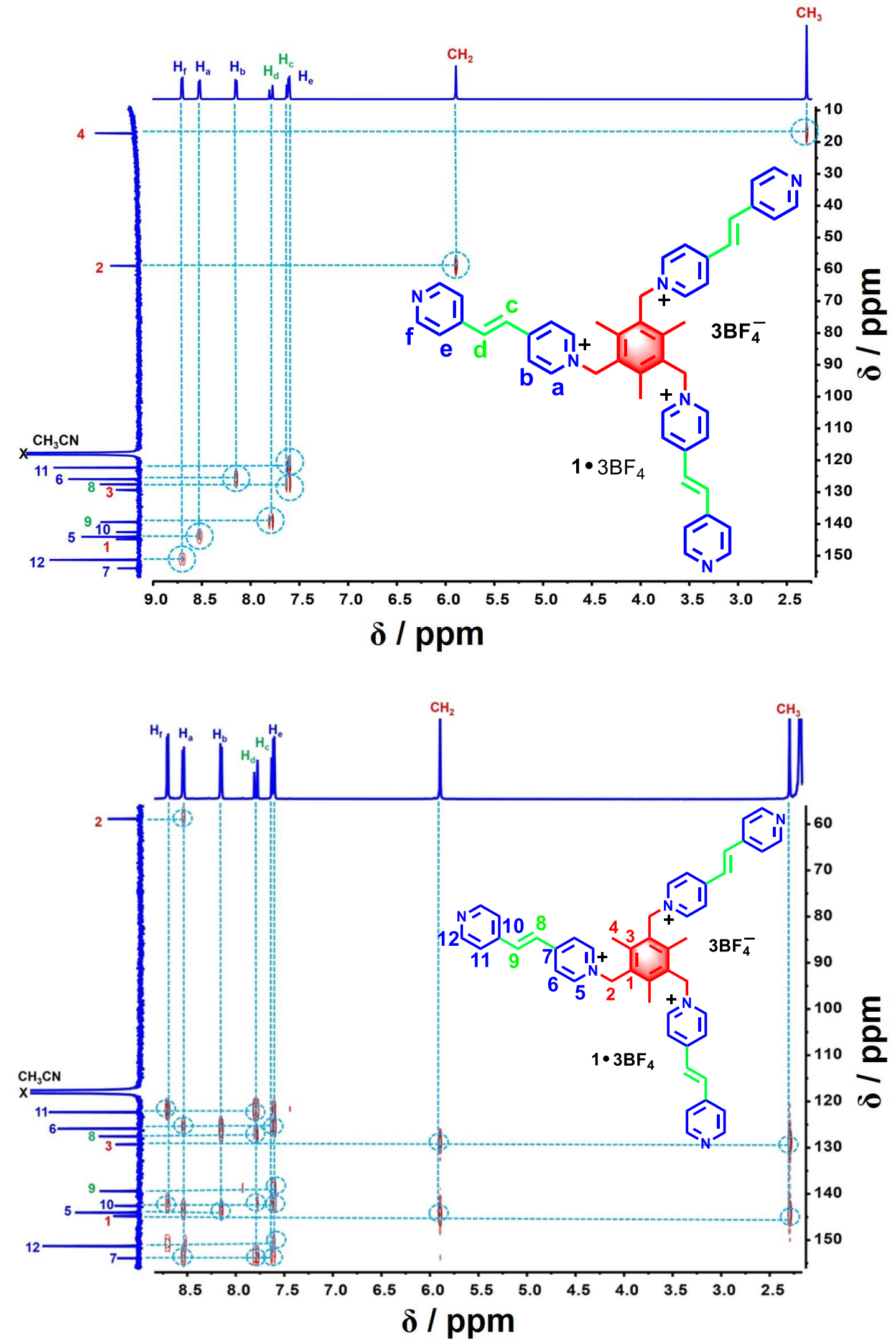

Figure S40. HSQC (top) and HMBC (bottom) NMR spectra of $1 \bullet 3 \mathrm{BF}_{4}$. 


\section{Section M. References}

1. E. R. Johnson, S. Keinan, P. Mori-Sanchez, J. Contreras-Garcia, A. J. Cohen, W. Yang, Revealing noncovalent interactions. J Am Chem Soc 132, 6498-6506 (2010).

2. Gaussian 16, Revision B.01, M. J. Frisch, G. W. Trucks, H. B. Schlegel, G. E. Scuseria, M. A. Robb, J. R. Cheeseman, G. Scalmani, V. Barone, G. A. Petersson, H. Nakatsuji, X. Li, M. Caricato, A. V. Marenich, J. Bloino, B. G. Janesko, R. Gomperts, B. Mennucci, H. P. Hratchian, J. V. Ortiz, A., F. Izmaylov, J. L. Sonnenberg, D. Williams-Young, F. Ding, F. Lipparini, F. Egidi, J. Goings, B. Peng, A. Petrone, T. Henderson, D. Ranasinghe, V., G. Zakrzewski, J. Gao, N. Rega, G. Zheng, W. Liang, M. Hada, M. Ehara, K. Toyota, R. Fukuda, J. Hasegawa, M. Ishida, T. Nakajima, Y. Honda, O. Kitao, H. Nakai, T. Vreven, K. Throssell, J. A. Montgomery, Jr., J. E. Peralta, F. Ogliaro, M. J. Bearpark, J. J. Heyd, E. N. Brothers, K. N. Kudin, V. N. Staroverov, T. A. Keith, R. Kobayashi, J. Normand, K. Raghavachari, A. P. Rendell, J. C. Burant, S. S. Iyengar, J. Tomasi, M. Cossi, J. M. Millam, M. Klene, C. Adamo, R. Cammi, J. W. Ochterski, R. L. Martin, K. Morokuma, O. Farkas, J. B. Foresman, and D. J. Fox, Gaussian, Inc., Wallingford CT, 2016.

3. H. S. Yu, X. He, S. L. Li, D. G. Truhlar, MN15: A Kohn-Sham global-hybrid exchange-correlation density functional with broad accuracy for multi-reference and single-reference systems and noncovalent interactions. Chem Sci 7, 5032-5051 (2016).

4. F. Weigend, R. Ahlrichs, Balanced basis sets of split valence, triple zeta valence and quadruple zeta valence quality for $\mathrm{H}$ to $\mathrm{Rn}$ : Design and assessment of accuracy. 
Phys Chem Chem Phys 7, 3297-3305 (2005).

5. O. V. Dolomanov, L. J. Bourhis, R. J. Gildea, J. A. Howard, H. Puschmann, OLEX2: A complete structure solution, refinement and analysis program. J. Appl. Cryst. 42, 339-341 (2009).

6. G. Sheldrick, SHELXT-Integrated space-group and crystal-structure determination. Acta Cryst. 71, 3-8 (2015).

7. G. Sheldrick, A short history of SHELX. Acta Cryst. 64, 112-122 (2008).

8. R. Dovesi et al., Quantum-mechanical condensed matter simulations with CRYSTAL. Wires Comput Mol Sci 8, e1360 (2018).

9. A. D. Becke, Density-functional thermochemistry. III. The role of exact exchange. J. Chem. Phys. 98, 5648-5652 (1993).

10. P. J. Stephens, F. J. Devlin, C. F. Chabalowski, M. J. Frisch, Ab initio calculation of vibrational absorption and circular dichroism spectra using density functional force fields. J. Phys. Chem. 98, 11623-11627 (1994).

11. C. T. Lee, W. T. Yang, R. G. Parr, Development of the Colle-Salvetti correlationenergy formula into a functional of the electron density. Phys. Rev. B 37, 785-789 (1988).

12. S. Grimme, J. Antony, S. Ehrlich, H. Krieg, A consistent and accurate ab initio parametrization of density functional dispersion correction (DFT-D) for the 94 elements H-Pu. J. Chem. Phys. 132, 154104 (2010).

13. J. P. Perdew, K. Burke, M. Ernzerhof, Generalized gradient approximation made simple. Phys. Rev. Lett. 77, 3865-3868 (1996).

14. S. Grimme, J. Antony, S. Ehrlich, H. Krieg, A consistent and accurate ab initio parametrization of density functional dispersion correction (DFT-D) for the 94 
elements H-Pu. J. Chem. Phys. 132, 154104 (2010).

15. M. R. Ryder, L. Dona, J. G. Vitillo, B. Civalleri, Understanding and controlling the dielectric response of metal-organic frameworks. ChemPlusChem 83, 308-316 (2018).

16. Q.-H. Guo, Z. Liu, P. Li, D. Shen, Y. Xu, M. R. Ryder, H. Chen, C. L. Stern, C. D. Malliakas, X. Zhang, et al., A hierarchical nanoporous diamondoid superstructure. Chem 5, 2353-2364 (2019).

17. M. Ryder, Physical phenomena in metal-organic frameworks: mechanical, vibrational, and dielectric response. University of Oxford (2017).

18. M. Cutini, B. Civalleri, M. Corno, R. Orlando, J. G. Brandenburg, L. Maschio, and P. Ugliengoa. J. Chem. Theory Comput. 12, 3340-3352 (2016).

19. D. Dubbeldam, S. Calero, D. E. Ellis, R. Q Snurr, RASPA: Molecular simulation software for adsorption and diffusion in flexible nanoporous materials. Mol. Simul., 42, 81-101 (2016).

20. J. Ilja Siepmann, D. Frenkel, Conflgurational bias monte carlo: A new sampling scheme for flexible chains. Mol. Phy., 75, 59-70 (1992).

21. S. L. Mayo, B. D. Olafson, W. A. Goddard III, DREIDING: A generic force field for molecular simulations. J. Phy. Chem., 94, 8897-8909 (1990).

22. W. L. Jorgensen, J. Chandrasekhar, J. D. Madura, R. W. Impey, M. L. Klein, Comparison of simple potential functions for simulating liquid water. J. Chem. Phy., 79, 926 (1983).

23. A. K. Rappé, W. A. Goddard III, Charge equilibration for molecular dynamics simulations. J. Phy. Chem., 95, 3358-3363 (1991) 